\title{
Impact of an Extruded Nucleotide on Cleavage Activity and Dynamic Catalytic Core Conformation of the Hepatitis Delta Virus Ribozyme
}

Jana Sefcikova, ${ }^{1}$ Maryna V. Krasovska, ${ }^{2}$ Nad'a Špačková, ${ }^{2}$ Jiří Šponer, ${ }^{2}$ Nils G. Walter ${ }^{1}$

${ }^{1}$ Department of Chemistry, Single Molecule Analysis Group, University of Michigan, 930 N. University Avenue,

Ann Arbor, MI 48109-1055

${ }^{2}$ Institute of Biophysics, Academy of Sciences of the Czech Republic, Královopolská 135, 61265 Brno, Czech Republic

Received 30 November 2006; revised 20 January 2007; accepted 20 January 2007

Published online 25 January 2007 in Wiley InterScience (www.interscience.wiley.com). DOI 10.1002/bip.20693

\section{ABSTRACT:}

The self-cleaving hepatitis delta virus (HDV) ribozyme is essential for the replication of HDV, a liver disease causing pathogen in humans. The catalytically critical nucleotide C75 of the ribozyme is buttressed by a trefoil turn pivoting around an extruded G76. In all available crystal structures, the conformation of G76 is restricted by stacking with G76 of a neighboring molecule. To test whether this crystal contact introduces a structural perturbation into the catalytic core, we have analyzed $\sim 200$ ns of molecular dynamics (MD) simulations. In the absence of crystal packing, the simulated G76 fluctuates between several conformations, including one wherein $G 76$ establishes a perpendicular base quadruplet

Correspondence to: Nils G. Walter; e-mail: nwalter@umich.edu or Jiři Šponer; e-mail: sponer@ncbr.chemi.muni.cz

Contract grant sponsor: $\mathrm{NIH}$

Contract grant number: GM62357

Contract grant sponsor: Wellcome Trust

Contract grant number: GR067507

Contract grant sponsor: Grant Agency of the Czech Republic

Contract grant numbers: GA203/05/0388, GA203/05/0009

Contract grant sponsor: Grant Agency of the Academy of Sciences of the Czech Republic

Contract grant number: 1QS500040581

Contract grant sponsor: Ministry of Education of the Czech Republic

Contract grant number: LC512

Contract grant sponsors: Ministry of Education of the Czech Republic; Margaret and Herman Sokol International Summer Research Fellowship Fund of the University of Michigan; NATO Science Fellowship Fund; Center for the Education of Women Sarah Winans Newman Scholarship Fund, Eli Lilly Predoctoral Fellowship Fund

\section{(\$)WILEY}

\section{InterScience}

(C) 2007 Wiley Periodicals, Inc. in the major groove of the adjacent P1 stem. Second-site mutagenesis experiments suggest that the identity of the nucleotide in position 76 (N76) indeed contributes to the catalytic activity of a trans-acting HDV ribozyme through its capacity for hydrogen bonding with P1. By contrast, in the cis-cleaving genomic ribozyme the functional relevance of N76 is less pronounced and not correlated with the P1 sequence. Terbium(III) footprinting and additional MD show that the activity differences between N76 mutants of this ribozyme are related instead to changes in average conformation and modified cross-correlations in the trefoil turn. (C) 2007 Wiley Periodicals, Inc. Biopolymers 85: 392-406, 2007. Keywords: conformational dynamics; hepatitis delta virus; molecular dynamics simulation; RNA quadruplet; terbium footprinting

This article was originally published online as an accepted preprint. The "Published Online" date corresponds to the preprint version. You can request a copy of the preprint by emailing the Biopolymers editorial office at biopolymers@wiley.com.

\section{INTRODUCTION}

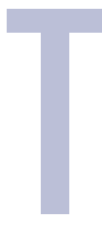

he hepatitis delta virus (HDV) is an unusual subviral pathogen and satellite of hepatitis B virus associated with chronic hepatitis. ${ }^{1}$ The HDV virion contains a single-stranded circular RNA genome that replicates through a double-rolling circle mechanism, generating multimeric genomic and antigenomic HDV RNAs. Both RNAs are autocatalytic and contain the HDV ribozyme motif 

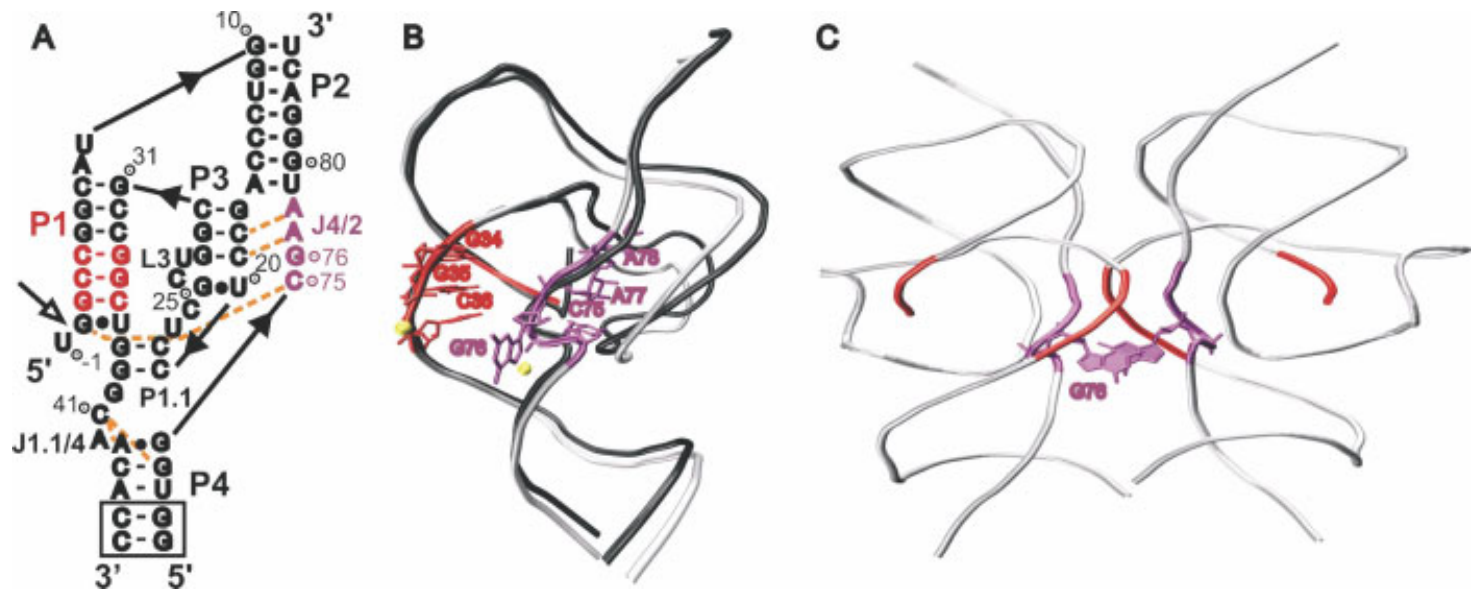

FIGURE 1 Overview of the precursor and $3^{\prime}$ product forms of the cis-acting genomic HDV ribozyme. (A) Sequence and secondary structure of the simulated HDV ribozyme, with the relevant part of the P1 stem and the trefoil turn color-coded. The product form lacks the U-1 nucleotide. Open arrow, cleavage site; rectangular box, two base pairs truncated in all MD simulations coded with "Trc" in their name; orange dashed lines, relevant tertiary interactions discussed in the text. (B) Overlap of the crystal structures of the reaction precursor (PDB ID $1 S J 3)^{9}$ in black and product $(\mathrm{PDB} \text { ID } 1 \mathrm{CX} 0)^{11}$ in silver with key nucleotides color-coded as in Panel A. Yellow spheres represent the positions of two relevant $\mathrm{Mg}^{2+}$ ions close to J4/2 in the product crystal structure. (C) Crystal lattice contact involving G76 base stacking between two symmetry-related HDV ribozyme molecules.

that catalyzes self-cleavage of the multimeric intermediates into monomeric RNA strands and their subsequent ligation into circular structures. The genomic and antigenomic ribozyme forms function in vitro at a minimal size of 85 nucleotides $^{2,3}$ and have very similar sequences that form closely related secondary structures. ${ }^{3-6}$ Site-specific self-(or cis-) cleavage entails nucleophilic attack by the adjacent, base-activated $2^{\prime}$-oxygen on the cleavage site phosphorus to substitute the acid-activated $5^{\prime}$-oxygen, generating $5^{\prime}$ - and $3^{\prime}$-cleavage products with $2^{\prime}, 3^{\prime}$-cyclic phosphate and $5^{\prime}$-hydroxyl termini, respectively. ${ }^{7}$ To accomplish the significant rate enhancement of $10^{7}$-fold over background, the HDV ribozyme utilizes multiple catalytic strategies, including control by a conformational switch ${ }^{8,9}$ and the first established example of general acid-base catalysis by an RNA side chain, cytosine 75 (C75). ${ }^{10}$

The three-dimensional structures of both precursor and $3^{\prime}$ product forms of the cis-acting genomic HDV ribozyme have been determined by X-ray crystallography to atomic resolution and, despite notable differences, share many common features. ${ }^{9,11,12}$ The overall structure is a nested double pseudoknot comprised of five Watson-Crick base-paired stems $\mathrm{P} 1, \mathrm{P} 1.1, \mathrm{P} 2, \mathrm{P} 3$, and $\mathrm{P} 4$, arranged in two coaxial stacks, P1|P1.1IP4 and P2IP3, which are connected by joining sequences (Figure 1A). The ribozyme's catalytic pocket is buried between the two stacks; it shows the essential cytosine 75 located proximal to the cleavage site, in a position where it has been proposed to act as either general base or general acid in the reaction mechanism. ${ }^{9,13-15}$ C75 is positioned deeply within the catalytic cleft by a tight trefoil turn that is characterized by an inversion in the orientation of the C75 and G76 riboses relative to the backbone direction and an extrusion of the G76 base into solvent (Figures 1B and 1C). In all crystal structures, two G76 bases from neighboring molecules stack to form a stabilizing crystal contact that buries $\sim 40 \AA^{2}$ surface area per guanine (Figure 1C). ${ }^{11}$ This latter contact is unique to the crystal packing, raising the immediate questions of (i) what alternate conformation(s) G76 may adopt in solution and (ii) how the nature of these conformations may impact catalysis. Previous mutagenesis studies of the cis-cleaving genomic HDV RNA have found that a G76U variant is 17 -fold less active than the wild-type (wt). ${ }^{16}$ Similarly, in the trans-acting HDV ribozyme, which utilizes an external RNA substrate but structurally behaves similarly to the cis-acting form, ${ }^{8,17-19}$ the same mutation results in a threefold decrease in cleavage activity, while a G76A mutation lowers activity fivefold. ${ }^{17}$ These previous observations suggest that position 76 is important for function of the HDV ribozyme.

To provide insight into realistic conformational dynamics in the absence of crystal lattice contacts, we have performed molecular dynamics (MD) simulations of the HDV ribozyme in the presence of explicit solvent and charge-screening cations (Figure 2). ${ }^{20,21}$ In a simulation of the genomic HDV ribozyme cleavage product, our trajectory analysis revealed a rapid rotation of G76 towards the catalytic pocket, followed by the formation and long-term retention of three hydrogen 

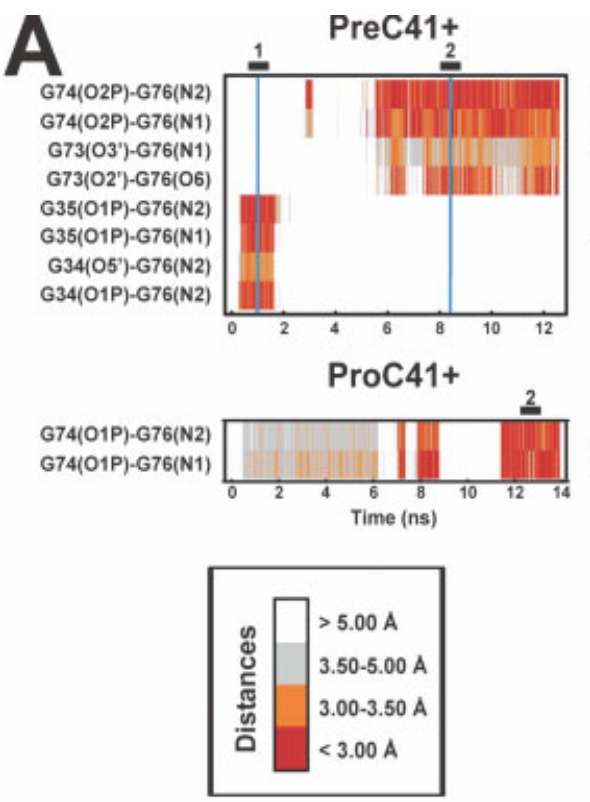

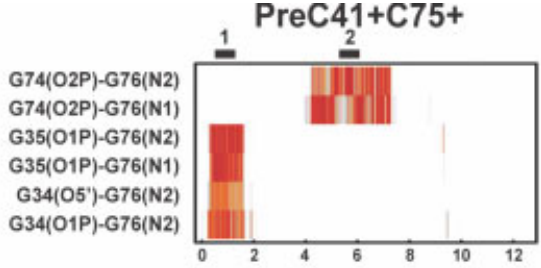

ProC41+C75+(1)
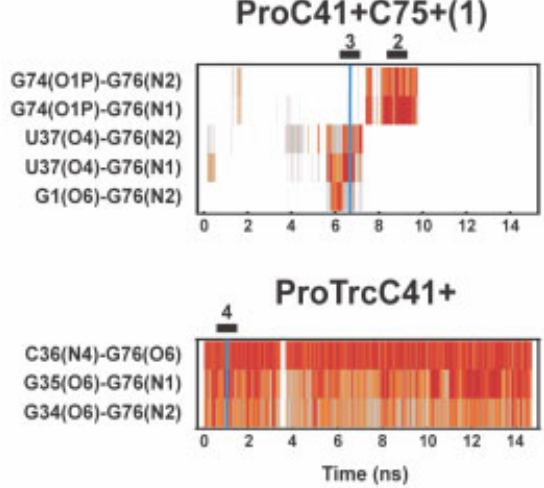
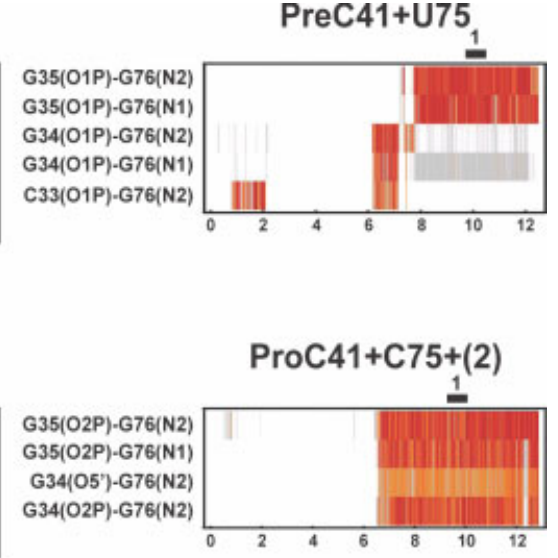

B

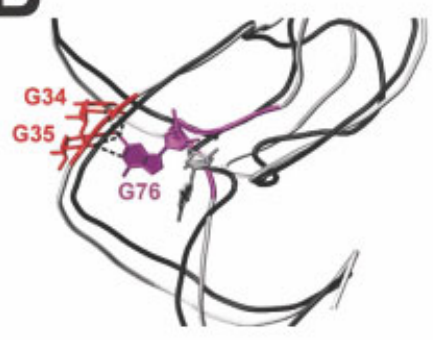

PreC41+ Conformation 1

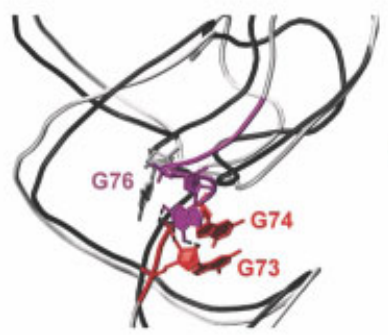

PreC41+ Conformation 2

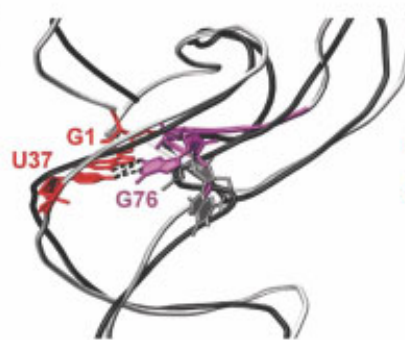

ProC41+C75+(1)

Conformation 3

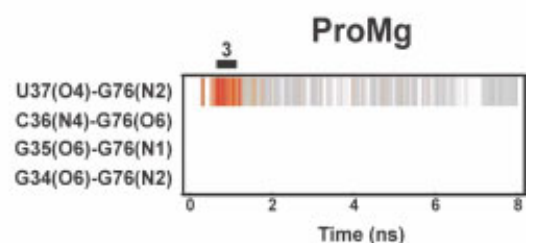

FIGURE 2 Broad structural dynamics of nucleotide G76 in our precursor and product MD simulations. ${ }^{20}$ (A) Time trajectories of relevant heavy-atom distances shown as list density plots over the entire simulation time. The same color scale for distances is used throughout the figures. A line in cyan highlights the time period used to obtain the representative average Conformations 1-4 shown in Panel B; vertical bars indicate all time periods where these conformations were observed. (B) Stick models showing an overlay of the crystal structure (in silver) and the representative average conformation (in black and color) obtained from the simulations in Panel A. Key nucleotides are color-coded as in Figure 1A; dashed black lines, H-bonds in the simulated conformation.

bonds with G34, G35, and C36 in the major groove of stem P1 (Figure 3). The establishment of such a perpendicular, Gspecific, and stable tertiary "docking" interaction involving an unusual base quadruplet suggests a mechanism by which nucleotide 76 (N76) may contribute to the conformational stability of the active site and exert the observed influence on catalytic activity.

To experimentally test for the plausibility of such hydrogen-bonding interactions between G76 and the major groove of stem $\mathrm{P} 1$, we performed second-site mutagenesis experiments. We find that the deleterious G76A mutation in a trans-acting HDV ribozyme is compensated for by mutations in P1 that complement the hydrogen-bonding pattern of adenosine, supporting a functional role for a docking interaction between N76 and P1 in this HDV ribozyme. By contrast, the cis-acting genomic HDV ribozyme does not show such a compensatory effect under a variety of buffer conditions. In fact, we find that, in contrast to the previous report, ${ }^{16}$ the relative activities of G76 mutants of the cis-acting genomic ribozyme follow the order $\mathrm{U} 76>\mathrm{G} 76 \approx \mathrm{A} 76>$ C76, inconsistent with a P1 interaction. To understand this relative reactivity of the N76 mutants of the cis-acting genomic ribozyme, we performed terbium(III)-mediated footprinting assays, which indeed detect conformational differences within the trefoil motif. We therefore carried out a number of additional mutant simulations (Table I), which 

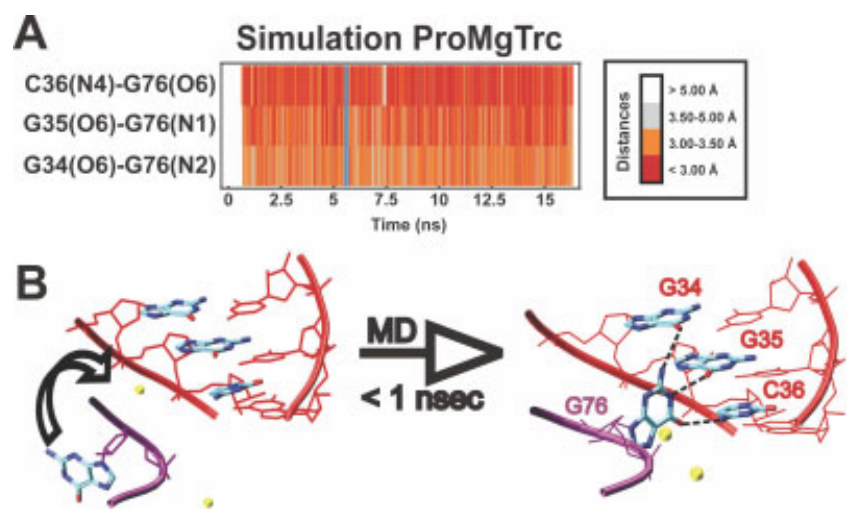

FIGURE 3 Structural dynamics of G76 in product MD simulation ProMgTrc. (A) Time trajectories of heavy-atom distances over the entire simulation time shown as list density plots. The same color scale for distances is used throughout the figures. The cyan vertical line represents the time period 5.7-5.8 ns of the simulation, for which an average structure was calculated to generate the stick model in Panel B. (B) Stick model, color-coded as in Figure 1A, showing the rotation of G76 from its starting geometry towards the major groove of the P1 stem over the first nanosecond of simulation ProMgTrc. Yellow spheres, relevant $\mathrm{Mg}^{2+}$ ions close to J4/2; broken line, H-bonds.

generate snapshots of plausible hydrogen-bonding interactions between N76 and the remainder of the ribozyme and suggest that not only the average structure, but also the structural dynamics of the trefoil turn change in response to N76 mutation. Given the role of the trefoil motif in positioning the catalytic C75, we suggest that these dynamic structural differences may explain the distinct reactivities of the G76 mutants of the cis-acting genomic HDV ribozyme.

\section{RESULTS}

\section{G76 Samples a Large Conformational Space and Forms a Long-Residence Perpendicular Base Quadruplet with Stem P1 in MD Simulations} We recently have described a total of $\sim 200$ ns of multiple explicit solvent MD simulations based on the crystal structures of the precursor and product forms of the genomic HDV ribozyme (Figure 1A). ${ }^{20,21}$ All simulations exhibited stable time trajectories in which the five double-helical regions $\mathrm{P} 1, \mathrm{P} 1.1, \mathrm{P} 2, \mathrm{P} 3$, and $\mathrm{P} 4$ remain relatively close to their starting crystal structures, resulting in low overall rootmean-square deviations. ${ }^{20,21}$ This is in agreement with the compact, closely interwoven, double-nested pseudoknot fold and extensive network of hydrogen bonding and stacking interactions of the HDV ribozyme, a large majority of which, yet not all, are preserved in our MD simulations. ${ }^{20}$

We find an unusually mobile nucleotide in G76 of the catalytic trefoil motif, which packs against G76 of a symmetryrelated HDV ribozyme in all of the dozen available crystal structures of the reaction precursor and product (Figure 1)., ${ }^{911,12}$ Partial solvent exposure of N76 is indeed consistent with our observations from 2-aminopurine fluorescence assays. ${ }^{17,22}$ In our nanosecond MD simulations, G76 samples a large conformational space, dynamically fluctuating between several weakly stabilized conformations. Figure $2 \mathrm{~B}$ illustrates the more frequently populated conformations of G76 sampled at various times during the simulations shown in Figure 2A. These conformations include base-backbone interactions with G72 and G74 in stem P4, base-backbone interactions with C33, G34, and G35 in stem P1, and base-base hydrogen

Table I Overview of Additional MD Simulations Carried Out in This Study (See Refs. 19 and 20 for Lists of Our Earlier Simulations)

\begin{tabular}{|c|c|c|c|c|c|}
\hline Simulation & $\begin{array}{l}\text { Starting } \\
\text { Structure }\end{array}$ & $\begin{array}{l}\text { Number of } \\
\text { Nucleotides }\end{array}$ & $\begin{array}{l}\text { Protonation } \\
\text { State of C41 }\end{array}$ & Duration (ns) & Presence of Ions \\
\hline ProMgTrcSim-A76 & Product $^{\mathrm{a}}$ & $58^{\mathrm{b}}$ & $\mathrm{C} 41$ & 12.5 & $38 \mathrm{Na}^{+}$and $9 \mathrm{Mg}^{2+c}$ \\
\hline ProMgTrcSim-C76 & Product $^{\mathrm{a}}$ & $58^{\mathrm{b}}$ & C41 & 15.5 & $38 \mathrm{Na}^{+}$and $9 \mathrm{Mg}^{2+\mathrm{c}}$ \\
\hline ProMgTrcSim-U76 & Product $^{\mathrm{a}}$ & $58^{\mathrm{b}}$ & $\mathrm{C} 41$ & 14 & $38 \mathrm{Na}^{+}$and $9 \mathrm{Mg}^{2+\mathrm{c}}$ \\
\hline $\operatorname{ProMgTrc}(2)$ & Product $^{\mathrm{d}}$ & $58^{\mathrm{b}}$ & $\mathrm{C} 41$ & 16.5 & $38 \mathrm{Na}^{+}$and $9 \mathrm{Mg}^{2+\mathrm{c}}$ \\
\hline ProMgTrc-A76 & Product $^{\mathrm{d}}$ & $58^{\mathrm{b}}$ & C41 & 16.5 & $38 \mathrm{Na}^{+}$and $9 \mathrm{Mg}^{2+\mathrm{c}}$ \\
\hline ProMgTrc-C76 & Product $^{\mathrm{d}}$ & $58^{\mathrm{b}}$ & $\mathrm{C} 41$ & 16.5 & $38 \mathrm{Na}^{+}$and $9 \mathrm{Mg}^{2+\mathrm{c}}$ \\
\hline ProMgTrc-U76 & Product $^{\mathrm{d}}$ & $58^{\mathrm{b}}$ & $\mathrm{C} 41$ & 16.5 & $38 \mathrm{Na}^{+}$and $9 \mathrm{Mg}^{2+\mathrm{c}}$ \\
\hline ProC41+A76 & Product $^{\mathrm{d}}$ & 62 & $\mathrm{C} 41 \mathrm{H}^{+}$ & 12.5 & $41 \mathrm{Na}^{+}$and $9 \mathrm{Mg}^{2+\mathrm{c}}$ \\
\hline ProC41+C76 & Product $^{\mathrm{d}}$ & 62 & $\mathrm{C} 41 \mathrm{H}^{+}$ & 13 & $41 \mathrm{Na}^{+}$and $9 \mathrm{Mg}^{2+\mathrm{c}}$ \\
\hline ProC41+U76 & Product $^{\mathrm{d}}$ & 62 & $\mathrm{C}_{4} 1 \mathrm{H}^{+}$ & 10 & $41 \mathrm{Na}^{+}$and $9 \mathrm{Mg}^{2+\mathrm{c}}$ \\
\hline
\end{tabular}

${ }^{\text {a }}$ Started from the average structure at $\sim 7.5$ ns of the earlier simulation ProMgTrc. ${ }^{20}$

${ }^{\mathrm{b}}$ In these simulations, two G-C Watson-Crick base pairs were truncated from the P4 stem (Figure 1A), which still led to stable simulations.

${ }^{\mathrm{c}}$ Nine $\mathrm{Mg}$ cations were placed initially as found in the starting product crystal structure, PDB ID 1CX0. ${ }^{11}$

${ }^{\mathrm{d}}$ Self-cleaved product crystal structure, PDB ID 1CX0. ${ }^{11}$ 
(H-)bonds with G1 and U37 in stem P1. It should be noted that none of these contacts caused any significant deformation of the P1 helix, J4/2, or the P4 backbone. Summarizing extensive analyses, our simulations suggest that under solution conditions G76 adopts a broad range of conformations typically very different from that found in the crystal structures, and that binding interactions of G76 in the major groove of stem P1 and to the backbone of stem P4 are generally the favored substates.

In a simulation starting from the product form of the genomic HDV ribozyme termed ProMgTrc (with C41 and C75 unprotonated and under inclusion of a truncated stem P4 and nine crystallographically resolved $\mathrm{Mg}^{2+}$ cations), we observed a rotation of G76 within 0.6 ns around its backbone from the extruded orientation towards the catalytic pocket $^{20,21}$ (Figure 3). Neither the conformations of the P1 stem nor the trefoil turn including the location of C75 are substantially perturbed from the starting crystal structure in response to this rotation (Figure 2B, Conformation 4), although the $\mathrm{C}_{4} 1 \mathrm{H}^{+}, \mathrm{A} 43, \mathrm{C} 44, \mathrm{G} 73$ quadruplet is lost (due to the lack of $\mathrm{C} 41$ protonation ${ }^{20}$ ), resulting in a slight bend of stem P4. The G76 rotation is accompanied by the formation and long-term retention of three H-bonds of G76 with the major groove of stem P1. More specifically, the H-bond between C36(N4) and G76(O6) has an occupancy of 59\% after its initial formation (where occupancy is defined as the fraction of time during which the $\mathrm{H}$-bond distance between the heavy atoms is $\leq 3.0 \AA$ and the H-bond angle is $\geq 120^{\circ}$ ), while that of the H-bond between G35(O6) and G76(N1) is $\sim 37 \%$, and that of the H-bond between G34(O6) and G76(N2) is $\sim 16 \%$ (Figure $3 \mathrm{~A}$ ). As a result, this "docked" conformation of G76 is retained for over 15 ns until the end of the simulation (Figure 3A). Therefore, while formation of this perpendicular quadruplet is a rather rare event (observed only in one of our 17 simulations ${ }^{20,21}$ ), the interaction is fairly stable once formed.

To test whether the newly formed tertiary contact of G76 is dependent on the presence of two proximal divalent metal ions from the starting crystal structure that remain stably bound (yellow spheres in Figure 3B), we took the coordinate set at $\sim 7.5 \mathrm{~ns}$ from simulation ProMgTrc and used it as the initial structure for a simulation wherein all $\mathrm{Mg}^{2+}$ ions were removed and $\mathrm{Na}^{+}$ions added instead to neutralize the system. We found that all H-bonds between G76 and G34, G35, and $\mathrm{C} 36$ remained unchanged for the total additional simulation time of $\sim 15 \mathrm{~ns}$ (data not shown), with similar conformational dynamics as observed in the parent simulation ProMgTrc. We conclude that "docking" of G76 with the P1 stem is a plausible long-lived substate of the HDV ribozyme that does not require $\mathrm{Mg}^{2+}$ ions. The existence of this substate is a hypothesis that lends itself to experimental testing.

Second-Site Mutagenesis Suggests a Functional Role for the Tertiary Interactions Between N76 and

Stem P1 in the Trans-Acting, but not the

Cis-Acting HDV Ribozyme

To enhance cleavage of a specific phosphodiester bond, a catalytic RNA must fold into a defined three-dimensional structure. Tertiary interactions contribute to the formation and stability of such an active conformation. To investigate the relevance of the computationally observed perpendicular base-base contacts between nucleotides G76 and G34, G35, C36 in the P1 helix, we performed second-site mutagenesis experiments on each a trans- and a cis-acting HDV ribozyme (Figures 4 and 5, respectively).

The trans-acting HDV ribozyme used for this study (Figure $4 \mathrm{~A}$ ) is a hybrid between the genomic and antigenomic forms that has been extensively characterized before and possesses catalytic properties at least at par with those of other trans-acting HDV ribozymes. ${ }^{8,17-19,22,23}$ Previously measured cleavage rate constants have demonstrated that mutation of G76 to A (G76A) in this ribozyme results in a fivefold decrease of cleavage activity, suggesting that it is a good model system for studying the functional relevance of G76. ${ }^{17}$ Its P1 helix sequence, however, is slightly different from that of the simulated genomic ribozyme. We therefore used the program InsightII to replace residues G34 and G35 (and G76) in the average structure of time period 5.7-5.8 ns of MD simulation ProMgTrc with A34 and C35 (and A76), respectively, of the trans-acting ribozyme sequence. This modeling predicts a single possible $\mathrm{H}$-bond between G76(O6) and C36(N4) in the wt compared with a possible A76(N1) and C35(N4) H-bond in the G76A mutant (Figure $4 \mathrm{~B})$. Importantly, substitution of an A2-U36 base pair into stem P1 predicts that the G76 wt loses its capacity for $\mathrm{H}$ bonding with the P1 stem altogether, while two contacts are predicted for the G76A mutant, namely A76(N6) with U36(O4), A76(N1) with C35(N4) (Figure 4C), suggesting this as a viable strategy to test for a functional N76:P1 interaction. If the interaction between N76 and the P1 stem plays a functional role, we predict that substituting an A2-U36 base pair for the isosteric G2-C36 in our trans-acting HDV ribozyme would reverse the activity loss of the G76A mutation.

Figure 4D shows that we indeed observed the predicted second-site reversion behavior. Cleavage rate constants were measured under single-turnover reaction conditions (in 40 $\mathrm{m} M$ Tris- $\mathrm{HCl}, \mathrm{pH} 7.5$, and $11 \mathrm{mM} \mathrm{MgCl}_{2}$ at $37^{\circ} \mathrm{C}$; Materials 


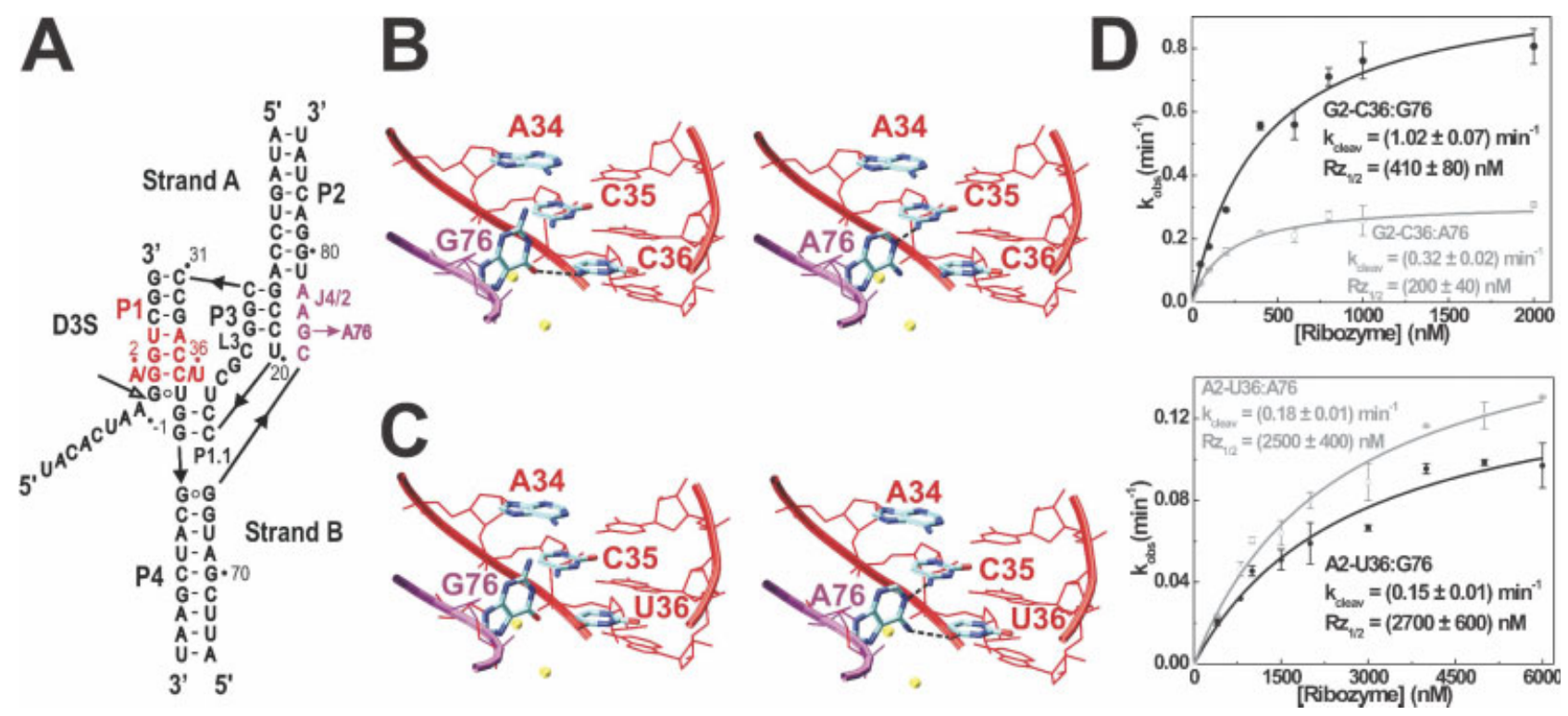

FIGURE 4 Second-site mutagenesis of the trans-acting HDV ribozyme. (A) Secondary structure of the trans-acting HDV ribozyme, indicating modifications introduced in this study at position 76 and at base pair 2-36 in the P1 stem. (B) Stick representations of the trans-acting ribozyme sequence, including either G76 or A76, modeled into the average structure from time period 5.75.8 ns of simulation ProMgTrc, showing N76 "docked" into the major groove of the P1 stem (see also Figure 3B). (C) Stick representations of the trans-acting ribozyme sequence, including an A2U36 base pair and either G76 or A76, modeled into the average structure from time period 5.7-5.8 ns of simulation ProMgTrc, showing N76 "docked" into P1. Note that Panels B and C represent structural models rather than MD simulation results. (D) Plots of the dependence of the observed rate constant $\left(\mathrm{k}_{\mathrm{obs}}\right)$ on the trans-acting HDV ribozyme concentration under standard conditions of $40 \mathrm{mM}$ Tris- $\mathrm{HCl}(\mathrm{pH} 7.5)$ and $11 \mathrm{mM} \mathrm{MgCl}_{2}$ at $37^{\circ} \mathrm{C}$. Data were fit with a simple binding equation (solid lines; see Materials and Methods) to derive the indicated cleavage activities at saturating ribozyme concentration $\left(k_{\text {cleav }}\right)$ and ribozyme half-titration points $\mathrm{Rz}_{1 / 2}$.
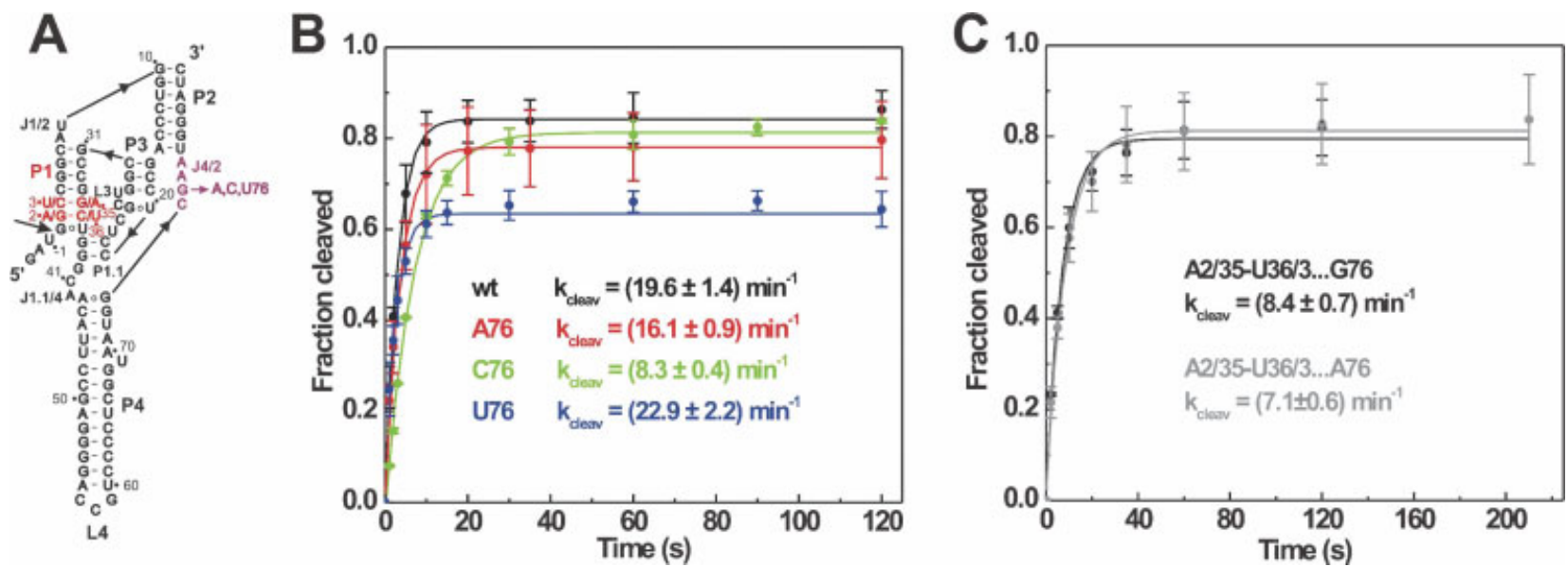

FIGURE 5 Cleavage assays of the cis-acting genomic HDV ribozyme. (A) Secondary structure of the cis-acting genomic HDV ribozyme, color-coded as in Figure 1A and indicating modifications introduced at position 76 and in stem P1. (B) Cleavage time courses of wt and G76 mutants under standard conditions of $11 \mathrm{mM} \mathrm{MgCl} 2$ at $22^{\circ} \mathrm{C}$ (see Materials and Methods). Data were fit with single-exponential increase functions (solid lines) to yield the rate constants $k_{\text {cleav }}$ (C) Cleavage time courses of wt and G76A mutant under standard conditions of $11 \mathrm{mM} \mathrm{MgCl}_{2}$ at $22^{\circ} \mathrm{C}$ (see Materials and Methods) in the presence of the P1 stem mutations described in Panel A. Data were fit with single-exponential increase functions (solid lines) to yield the rate constants $k_{\text {cleav }}$. 
and Methods), which consist of trace amounts of radiolabeled substrate with saturating ribozyme concentrations. In each case we performed a ribozyme titration to ensure that saturation is reached and slight differences in binding affinity of the substrates are compensated; the observed pseudo-firstorder rate constants $\left(k_{\mathrm{obs}}\right)$ were plotted as a function of ribozyme concentration and fit with a simple binding equation (Materials and Methods), yielding the cleavage rate constants $k_{\text {cleav }}$ reported in Figure 4D. The approximately threefold slower rate constant of the G76A mutant compared with the G76 wt in the presence of the wt G2-C36 base pair in stem $\mathrm{P} 1$ is similar to that reported previously. ${ }^{17}$ Importantly, the relative activities of G76 wt and G76A mutant are reversed in the presence of the A2-U36 carrying P1 over the whole ribozyme concentration range, supporting a weak, but noticeable functional role of the interactions between N76 and the major groove of the $\mathrm{P} 1$ stem in our trans-acting HDV ribozyme. Notably, the substrate binding affinity (expressed as the ribozyme half-saturation point $\mathrm{Rz}_{1 / 2}$, Figure $4 \mathrm{D}$ ) as well as the cleavage rate constant $k_{\text {cleav }}$ were considerably weakened and slowed, respectively, in both mutants that carry the thermodynamically less stable A2-U36 base pair in place of the wt G2-C36.

To more rigorously test the functional relevance of the N76-stem P1 interaction, we decided to also probe the cisacting genomic ribozyme, on which our MD simulations are based. We performed a similar structure prediction analysis using InsightII (data not shown), which found no contact between A76 and the wt P1 for the G76A mutant compared with the three H-bonds predicted for G76. Conversely, a quadruple mutation in the P1 stem of G2-C36 to A2-U36 and C3-G35 to U3-A35 is predicted to allow for two Hbonds with A76, but only one with G76, so that we chose this mutation (referred to as A2/35-U36/3) for our secondsite reversion experiment. Self-cleavage of the resulting four permuted cis-acting ribozymes follows first-order kinetics, with the cleavage rate constants and final cleavage extents reported in Table II. The kinetic parameters for our wt, $k_{\text {cleav }}=19.6 \mathrm{~min}^{-1}$ and final cleavage extent $=86 \%$ in the presence of $11 \mathrm{mM} \mathrm{MgCl} 2$ at $22^{\circ} \mathrm{C}$, are in good agreement with values reported previously for the genomic HDV ribozyme. $^{6}$ An only slightly, yet reproducibly lower cleavage rate constant of $16.1 \mathrm{~min}^{-1}$ was measured for the G76A mutant (Figure 5B, Table II), consistent with a rather weak functional relevance of the identity of N76, in contrast to an earlier report. ${ }^{16}$ Significantly, the rate constant measured for the A2/ 35-U36/3:A76 second-site mutant is still $\sim 1.2$-fold lower than that for the A2/35-U36/3 mutant with native G76 (Figure 5C, Table II), that is, we observe no second-site reversion. Recovery of activity by second-site mutagenesis was also not observed under less favorable reaction conditions, such as in the absence of prefolding of the ribozyme in annealing buffer, at lower $\mathrm{Mg}^{2+}$ concentration, or at higher $\mathrm{pH}$ (Table II), providing little evidence for a functional relevance of an interaction of N76 with the major groove of stem P1 in the cis-acting genomic HDV ribozyme even under high-pH conditions where the catalytically important protonated $\mathrm{C}_{41 \mathrm{H}^{+}}, \mathrm{A} 43, \mathrm{C} 44, \mathrm{G} 73$ quadruplet is less likely to form. ${ }^{24}$

In summary, self-cleavage of the genomic precursor ribozyme with varying N76 (Figure 5A) shows first-order kinetics with the cleavage rate constants following the trend: U76 > G76 $\approx$ A76 $>$ C76 (Figure 5B, Table II), in contrast to a previously estimated $\sim 17$-fold decrease in the cleavage rate constant for the G76U mutant. ${ }^{16}$ While we find the identity of N76 to have a moderate, reproducible, up to 2.4-fold effect on the kinetic behavior of the cis-acting genomic HDV ribozyme, no obvious correlation is found between catalytic activity and number of expected perpendicular $\mathrm{H}$-bonds of N76 with the major groove of P1 (see earlier and data not shown), in contrast to our findings for the trans-acting ribozyme. Notably, the extent of cleavage decreases slightly from $86 \%$ for the wt to $66 \%$ for the G76U mutant, which may be due to enhanced misfolding of the G76U mutant and may possibly have been misinterpreted as lower catalytic activity in the earlier study. ${ }^{16}$ We therefore set out to find other

Table II Rate Constant (in $\mathrm{min}^{-1}$ ) and Final Cleavage Extent of Wild Type and Mutants of the Cis-Acting Genomic HDV Ribozyme

\begin{tabular}{|c|c|c|c|c|c|c|}
\hline \multirow[b]{2}{*}{ Conditions } & \multicolumn{6}{|c|}{ Rate Constant $\left(\min ^{-1}\right)$} \\
\hline & Wild Type & G76A & G76C & G76U & $\mathrm{A} 2 / 35-\mathrm{U} 36 / 3$ & A2/35-U36/3:A76 \\
\hline $\mathrm{pH} 7.5,11 \mathrm{mM} \mathrm{Mg}{ }^{2+}$ & $19.6 \pm 1.4(86)^{\mathrm{a}}$ & $16.1 \pm 0.9(80)$ & $8.3 \pm 0.4(84)$ & $22.9 \pm 2.2(66)$ & $8.4 \pm 0.7(82)$ & $7.1 \pm 0.6(84)$ \\
\hline $\mathrm{pH} 9.5,11 \mathrm{mM} \mathrm{Mg}^{2+}$ & $4.6 \pm 0.4(79)$ & $2.2 \pm 0.1(73)$ & ND & ND & $1.6 \pm 0.4(88)$ & $0.8 \pm 0.2(91)$ \\
\hline $\mathrm{pH} 7.5,1 \mathrm{mM} \mathrm{Mg}^{2+}$ & $2.0 \pm 0.8(93)$ & $1.4 \pm 0.3(97)$ & ND & ND & $0.70 \pm 0.02(78)$ & $0.79 \pm 0.07(94)$ \\
\hline No annealing & $1.4 \pm 0.3(77)$ & $0.9 \pm 0.2(74)$ & ND & ND & $0.75 \pm 0.06(88)$ & $0.47 \pm 0.03(87)$ \\
\hline
\end{tabular}

${ }^{\mathrm{a}}$ Values within parentheses indicate the final cleavage extent (\%). 
factors that may mediate the observed moderate effect of N76 substitution on catalytic activity of the cis-acting genomic HDV ribozyme.

Terbium(III) Footprinting Shows That the Average Conformation of the Trefoil Turn in the Cis-Acting Genomic HDV Ribozyme is Influenced by the Identity of the N76 Base

To examine the impact of N76 on folding of the catalytic pocket, including the catalytically involved, immediately adjacent C75, we used terbium(III) footprinting on the more easily accessible products of the self-cleaving genomic N76 mutants. High (millimolar) concentrations of terbium(III) can be used to slowly cut an RNA phosphodiester backbone in a largely sequence-independent manner, preferentially in single-stranded or non-Watson-Crick base-paired regions, thus generating a footprint of the RNA's secondary and tertiary structure at nucleotide resolution. ${ }^{18,25-30}$ Trace amounts of the radiolabeled product forms of the cis-acting genomic HDV ribozyme with varying N76 were prefolded under standard conditions in the presence of $11 \mathrm{mM} \mathrm{Mg}^{2+}$, followed by addition of $1 \mathrm{mM} \mathrm{Tb^{3+ }}$ to initiate slow backbone scission at $37^{\circ} \mathrm{C}$ over $1 \mathrm{~h}$ (under these conditions, only a small fraction of RNA is cleaved, avoiding secondary hits on an already cut RNA molecule). The footprinting patterns of all four constructs showed extensive similarities with respect to their protected and susceptible regions, consistent with the fact that all constructs varied only in the N76 position. Moreover, all four scission patterns agreed with the expected secondary structure of the HDV ribozyme; all five WatsonCrick base-paired stems, P1-P4 and P1.1, were well protected, while strong scission was observed in the backbone of loops L3 and L4 as well as in joiners J1.1/4 and J4/2 (data not shown).

To assess any minor differences, band intensities were quantified directly from the sequencing gel (Figure 6). Significant and reproducible variations in backbone scission patterns among the N76 mutants were found exclusively in their J4/2 junction trefoil turns encompassing C75 through A78, while, for example, their adjacent U69 regions were indistinguishable (Figure 6B). The purine containing G76 wt and the G76A mutant show similar scission patterns with intensities following the order: $\mathrm{C} 75>76>$ A77 $>$ A78, i.e., the most intense scission was observed $3^{\prime}$ of C75. Pyrimidines in position 76 revealed distinct scission patterns from those of the purine mutants, following the intensity order A77 > C76 > $\mathrm{C} 75>\mathrm{A} 78$ in the case of $\mathrm{G} 76 \mathrm{C}$ and $\mathrm{U} 76 \approx \mathrm{C} 75>\mathrm{A} 77>\mathrm{A} 78$ in the case of $\mathrm{G} 76 \mathrm{U}$ (Figure $6 \mathrm{~B}$ ). Our findings thus provide evidence that folding of the trefoil turn depends on the iden-
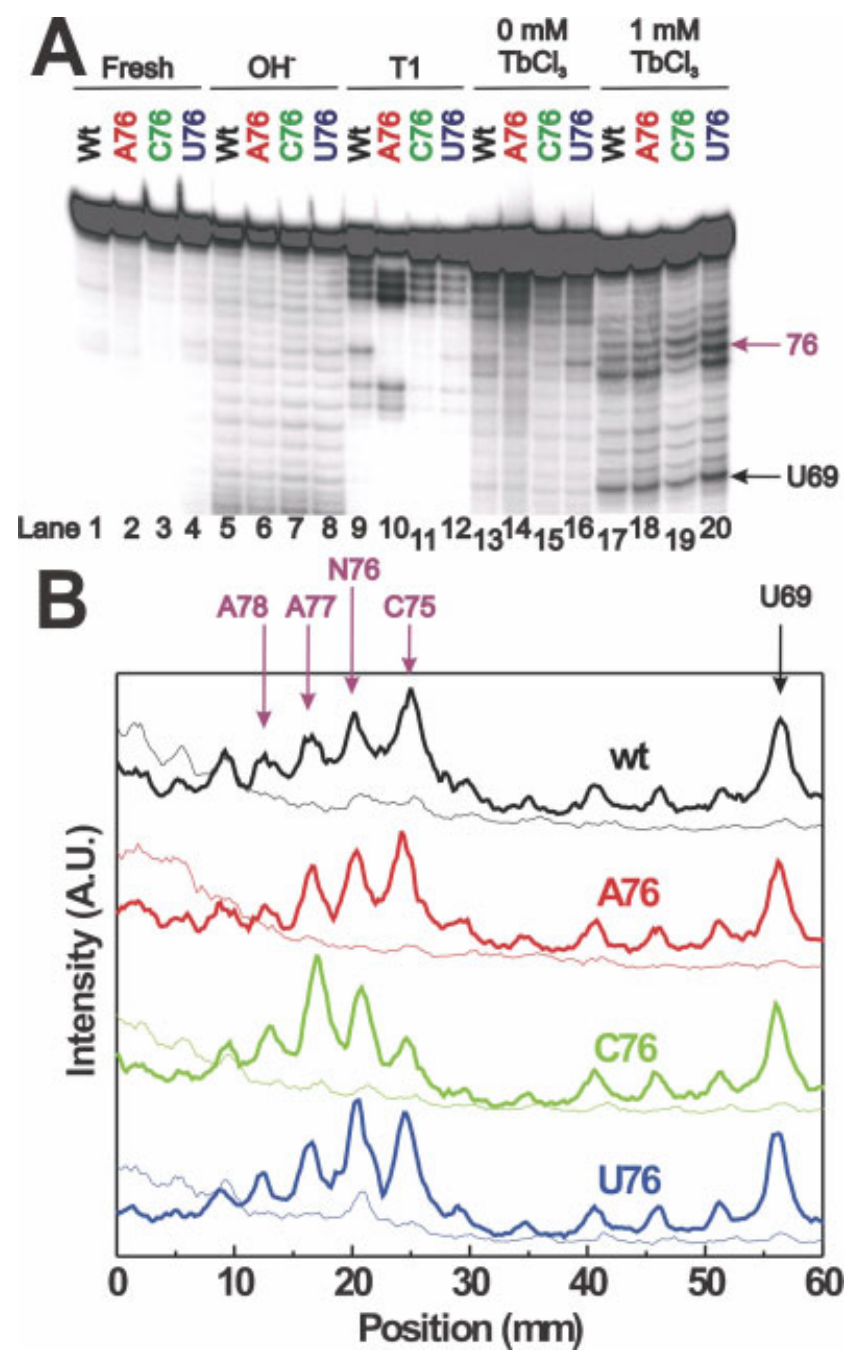

FIGURE 6 Terbium(III)-mediated footprinting of the $5^{\prime}-{ }^{32} \mathrm{P}-\mathrm{la}-$ beled product forms of the cis-acting genomic HDV ribozymes. (A) Sequencing gel showing the footprints of the product forms of the cis-acting ribozyme carrying N76 modification as indicated (see Materials and Methods). From left to right: Lanes 1-4, 5'-radiolabeling product without incubation (Fresh); Lanes 5-8, alkaline hydrolysis ladder $\left(\mathrm{OH}^{-}\right)$; Lanes 9-12, G-specific RNase T1 ladder; Lanes 13-16, samples incubated in the absence of terbium(III); Lanes 17-20, samples incubated in the presence of $1 \mathrm{mM} \mathrm{TbCl}_{3}$. (B) Quantification of terbium(III)-induced backbone scission from the gel in Panel A. Line plots show the relative scission intensity in arbitrary units (A.U.) $3^{\prime}$ of the indicated nucleotides. Thin lines, background in Lanes 13-16 of Panel A (RNA incubated in standard buffer without terbium).

tity of the N76 base, such that C76 and U76 cause average conformations distinct from those formed in the presence of G76 and A76. Notably, these structural data correlate with the fact that the pyrimidine containing mutants show the most distinct catalytic rate constants, decelerated for G76C $\left(8.3 \mathrm{~min}^{-1}\right)$, accelerated for G76U $\left(22.9 \mathrm{~min}^{-1}\right)$, when compared with the purine containing wt $\left(19.6 \mathrm{~min}^{-1}\right)$ and G76A 
$\left(16.1 \mathrm{~min}^{-1}\right.$ ) (Figure 5B, Table II). We propose that the conformational rearrangements we observe for N76 variants in the J4/2 region are correlated with catalytic function of the cis-acting genomic HDV ribozyme. Next, we sought to further support this hypothesis by MD simulating the N76 mutants.

\section{N76 Position Affects the Structural Dynamics of the Active Site}

To explain our footprinting results on the N76 mutants and obtain an atomistic survey of differences in their structural dynamics, we simulated the molecular dynamics of the four N76 mutants starting from the product crystal structure and substituting A, C, and U for G76. We performed these simulations, first, in the absence of $\mathrm{C} 41$ protonation, giving rise to simulations ProMgTrc(2) (which carries the wt G76 in a repetition of our original simulation ProMgTrc ${ }^{20,21}$ ), ProMgTrcA76 etc. (Table I); and, second, in the presence of a protonated $\mathrm{C}_{4} 1 \mathrm{H}^{+}$, giving rise to simulations ProC41+A76 etc. (Table I). Figure 7 summarizes the results. Nucleotide A76 behaves similarly in simulations ProMgTrc-A76 and ProC41+A76; it does not rotate towards the P1 stem and remains exposed to solvent, forming transient $\mathrm{H}$-bond interactions with G74(O1P) of the J4/2 backbone. Nucleotide C76 adopts distinct conformations in simulations ProMgTrc-C76 and ProC41+C76; only in the latter simulation it becomes oriented towards the P1 stem within the first 4 ns of simulation, where it is stabilized by interactions with G35(N7) and G35(O2P) for the rest of the trajectory. Nucleotide U76 stays mostly extruded into solution in both simulations ProMgTrc-U76 and ProC41+U76, with a close contact between $\mathrm{U} 76(\mathrm{~N} 3)$ and $\mathrm{G} 74(\mathrm{O} 1 \mathrm{P})$ of the J4/2 backbone. In the repeat simulation of the wild-type, $\operatorname{ProMg} \operatorname{Trc}(2)$, nucleotide G76 again turns towards the P1 stem within the first nanosecond of the simulation, but then establishes $\mathrm{H}$-bond interactions with $\mathrm{G} 35(\mathrm{O} 2 \mathrm{P})$ and $\mathrm{G} 34(\mathrm{O} 2 \mathrm{P})$ of the stem $\mathrm{P} 1$ backbone (similar to Conformation 1 in Figure $2 \mathrm{~B}$ ) and only a very transient H-bond between $\mathrm{C} 36(\mathrm{~N} 4)$ and G76(O6). This behavior contrasts with the extensive $\mathrm{H}$-bonds with the major groove of the P1 stem that the original ProMgTrc simulation established (Figure 7A). ${ }^{20}$ Simulation $\operatorname{ProMg\operatorname {Trc}}(2)$ did not reveal any change in the orientation of stem $\mathrm{P} 4$, as the base quadruplet at the top of $\mathrm{P} 4$, comprised of the unprotonated C41, A43, C44, and G73 (Figure 1A), stayed in a crystal-structure-like conformation through the association with long-residency $\mathrm{Na}^{+}$ions (data not shown). By comparison, the original ProMgTrc simulation, in the absence of C41 protonation and through the resultant loss of the C41, A43, C44, G73 quadruplet, developed a slight bend in stem P4, possibly facilitating the described long-residency triple $\mathrm{H}$ bond interaction of G76 with the bases in stem P1 (Figure 2B, Conformation 4). ${ }^{20,21}$ Nevertheless, nucleotide G76 in both simulations shows a significant tendency to establish stable $\mathrm{H}$-bond contacts with the P1 stem either through base-base (ProMgTrc) or base-backbone interactions (ProMgTrc(2)). Even though the presented MD simulations likely do not sample conformational space completely, we conclude that the dynamic behavior of the joiner sequence $\mathrm{J} 4 / 2$, which harbors the catalytic C75, significantly varies among the N76 mutants. This variation in dynamics and preferred substates likely gives rise to differences in average conformation, which themselves are computationally not accessible due to inadequate sampling but are consistent with our observations from terbium(III) footprinting.

To ask how the mutants behave dynamically when starting out in the conformation with N76 close to P1, we ran simulations starting from the average "docked" structure at $\sim 7.5$ ns of simulation ProMgTrc and substituted A, C, and U for G76 to yield simulations ProMgTrcSim-A76 etc. (Table I). As expected due to lack of complementarity, none of the mutants retained the base-base interactions of G76 with the major groove of the P1 stem. N76 in the mutants again adopted various dynamic conformations, either forming a transient $\mathrm{H}$-bond with the backbone of stems $\mathrm{P} 1$ or $\mathrm{P} 4$, or becoming solvent-exposed (Figure 7). Only G76 retained its "docked" conformation through base-base contacts with P1. Again, these observations suggest that there exist systematic differences in accessible substates for the N76 mutants.

Finally, to ask whether there may exist a direct correlation between N76 structural dynamics and catalytic function, we performed a cross-correlation analysis of all catalytic core nucleotides in our MD simulations. Figure 8 shows the representative cross-correlations found in the catalytic core of the wt simulation ProMgTrc and the mutant simulations ProC41+A76, ProC41+C76, and ProC41+U76, as indicated. The trefoil turn of junction J4/2 comprising C75 through A78 helps position the catalytic nucleotide C75 in close proximity to the scissile phosphate; more specifically, in the product crystal structure C75 makes a H-bond with the O5'-leaving group on $\mathrm{G} 1,{ }^{11,12}$ while in the precursor it appears to be hydrogen bonded to the scissile phosphate and attacking $2^{\prime}$ $\mathrm{OH}$ nucleophile. ${ }^{9,20}$ The trefoil turn is held in place by extrusion of G76, the stacking of G74 under P1.1, and the Aminor motif formed between A77/A78 and the minor groove of stem P3 (Figure 1A). No significant changes in the S-shape of the backbone of the trefoil turn were observed in our simulations, regardless of the orientation of N76. Our cross-correlation analysis reveals that the J4/2 nucleotides in all N76 variants move generally in a correlated fashion (i.e., synchro- 
A

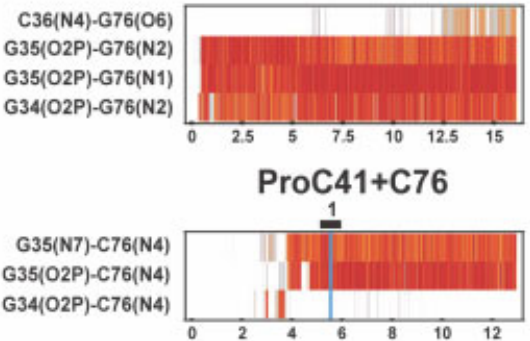

ProMgTrcSim-C76

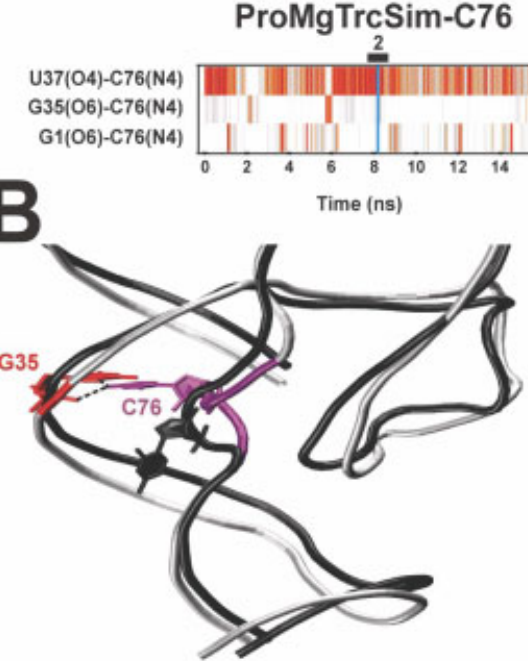

ProC41+Mg-C76

Conformation 1

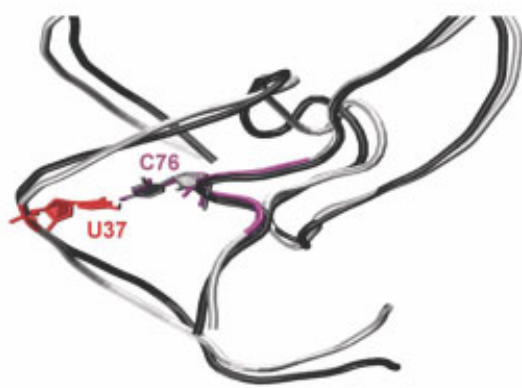

ProMgTrcSim-C76 Conformation 2
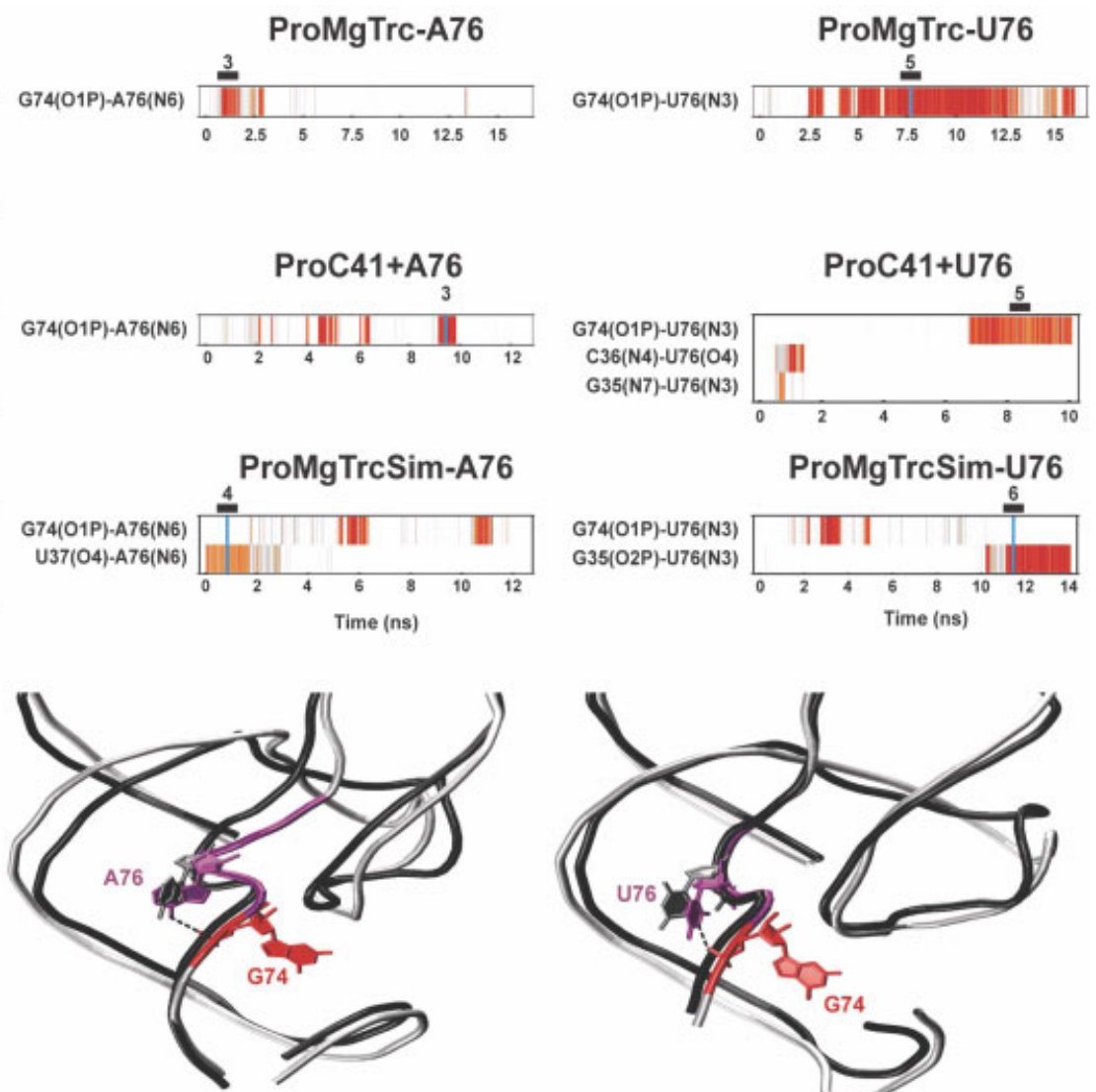

ProC41+Mg-A76
Conformation 3

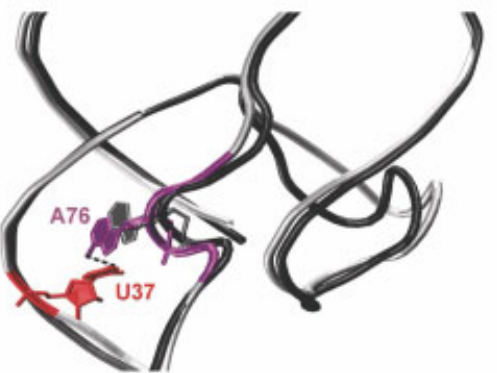

ProMgTrcSim-A76 Conformation 4

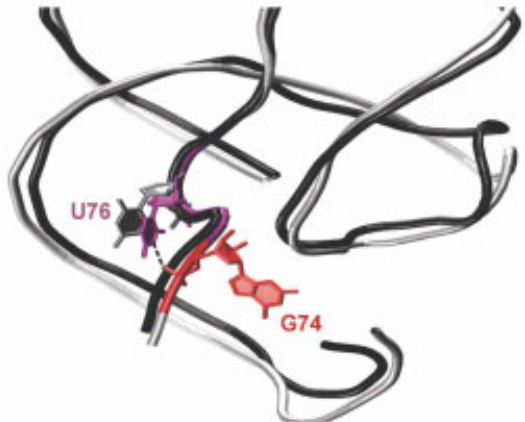

ProMgTrc-U76

Conformation 5

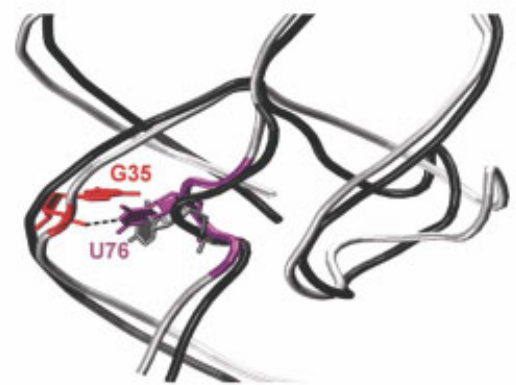

ProMgTrcSim-U76

Conformation 6

FIGURE 7 Structural dynamics of nucleotide N76 in product MD simulations with varying N76. (A) Time trajectories of heavy-atom distances shown as list density plots over the entire simulation time. The same color scale for distances as in Figures 2 and 5 is used. A line in cyan highlights the time period used to obtain the representative average Conformations 1-6 shown in Panel B; vertical bars indicate all time periods where these conformations were observed. (B) Stick models showing an overlay of the crystal structure (in silver) and the representative average conformation (in black and color) obtained from the simulations in Panel A. Key nucleotides are color-coded as in Figure 1A; dashed black lines, H-bonds in the simulated conformation.

nously in similar directions; Figure 8A). Notably, the least catalytically active mutant G76C shows the strongest catalytic core cross-correlations, while the most active mutant G76U shows the least cross-correlations (Figure 8A). This observation suggests that enhanced flexibility in the catalytic core may be correlated with enhanced catalytic activity. Such cor- 

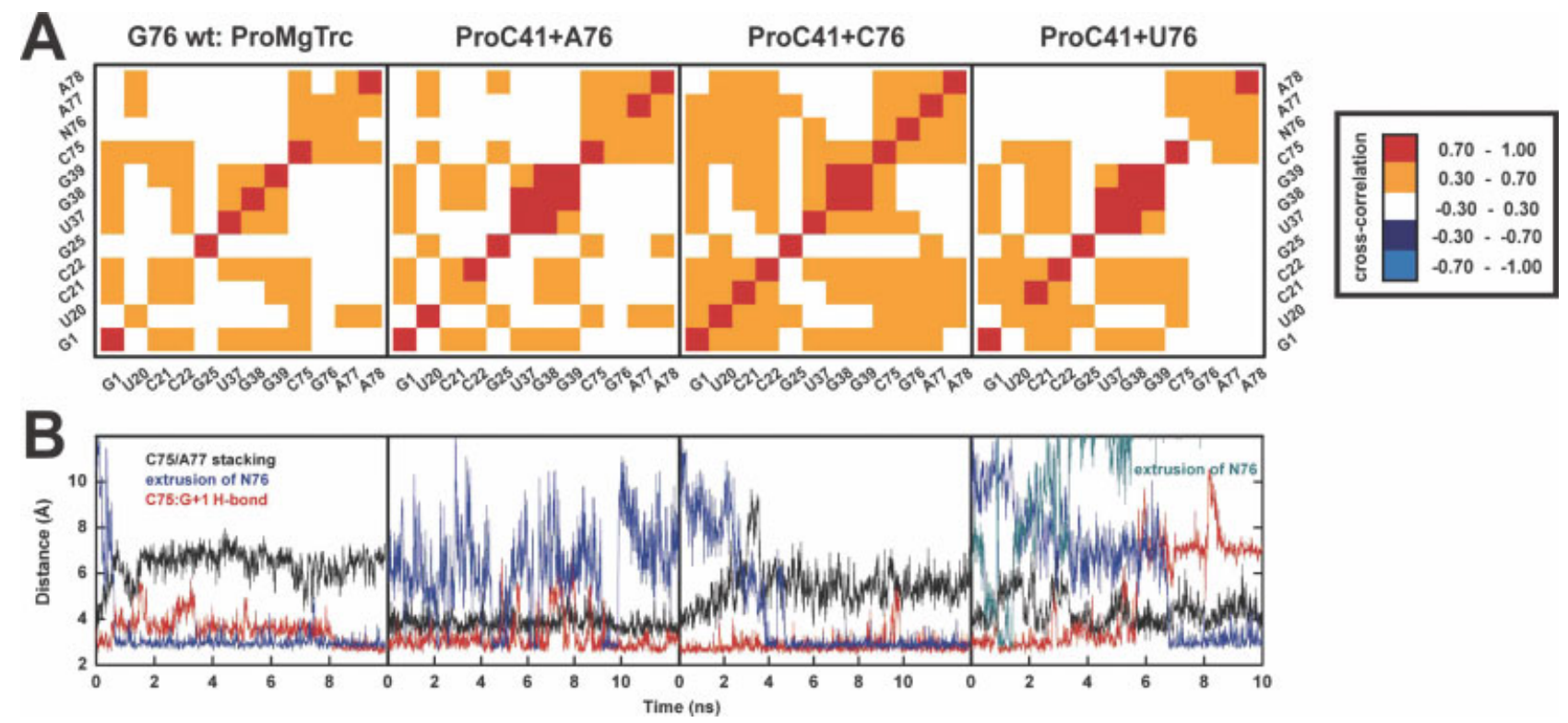

FIGURE 8 Structural dynamics of the active site depending on the identity of N76 in representative product MD simulations. (A) Cross-correlation maps of the nucleotides in the active site over the first 10 ns simulation time of four representative simulations as indicated. Positive correlations are highlighted in red and orange as described by the color code. (B) Time trajectories of important heavy-atom distances over the entire simulation time of the same simulations described in Panel A; C75/A77 stacking (black line) is represented by the distance C75(N3)-A77(N1); the catalytic Hbond (red line) is represented by the distance $\mathrm{G} 1\left(\mathrm{O}^{\prime}\right)-\mathrm{C} 75(\mathrm{O} 2)$; extrusion of nucleotide N76 (blue line) is represented by the distance C36(N4)-G76(O6) in simulation ProMgTrc, by the distance G74(O1P)-A76(N6) in simulation ProMgTrc-A76, by the distance G35(O2P)-C76(N4) in simulation ProMgTrc-C76, and by the distances G74(O1P)-U76(N3) (blue line) and C36(N4)-U76(O4) (cyan line) in simulation ProMgTrc-U76.

relation is corroborated by monitoring specific distances in the catalytic core, including the N76 extrusion away from stem P1, stacking of C75 and A77, and H-bonding between the catalytic C75 and G1 (Figure 8B). Generally, the more active G76 wt and U76 and A76 mutants are characterized by a more dynamic $\mathrm{C} 75: \mathrm{G}+1$ catalytic core $\mathrm{H}$-bond than the least active C76 mutant. We also attempted rather extensive Locally Enhanced Sampling (LES) simulations of the G76 nucleotide to increase conformational sampling and observe rare transitions. However, in contrast to our preceding study on Guanine quadruplex DNA loops,${ }^{31}$ LES did not provide a converging picture of the G76 dynamics, consistent with G76's ability to sample a large conformational space.

\section{DISCUSSION}

RNA molecules function only once they fold into their active conformation, which often can be captured in the static image provided by $\mathrm{X}$-ray crystallography. In addition, the activity of ribozymes often requires that specific regions have local structural flexibility and/or the ability to switch from one conformation to another. Understanding ribozyme func- tion thus demands an accurate and, ideally, comprehensive knowledge of the dynamics of the RNA's secondary and tertiary structure. An atomistic view of the structural dynamics as necessary for function of a ribozyme can only be obtained from few approaches, among them prominently MD simulations.

Our extensive MD simulations on both precursor and product forms of the cis-acting genomic HDV ribozyme ${ }^{20,21}$ show that in solution, in the absence of a $40-\AA^{2}$ crystal stacking contact, G76 exhibits significant structural dynamics and samples various conformations, as hinted at by a recent fluorescence study where 2 -aminopurine was substituted into the 76 position (AP76). ${ }^{22}$ Here, we extend our previous work to observe the conformational dynamics of G76 in atomistic detail and identify conformational substates of the catalytically essential trefoil turn that pivots around G76. Our MD simulations reveal a minimum of four specific conformational substates in which G76 remains at least partially solvent-exposed (Figure 2B), consistent with the fluorescence quenching observed in case of the AP76 substitution. All of these substates retain the basic S-shape of the trefoil turn. A particularly striking and stable conformation (Conformation 
4 in Figures 2B and 3B) is observed wherein G76 establishes an unusual perpendicular quadruplet interaction with bases in the major groove of the adjacent P1 stem (to our knowledge never before observed). Second-site mutagenesis of P1 suggests that H-bonding between N76 and P1 indeed plays a functional role in the catalytic activity of the trans-acting, but not the cis-acting genomic HDV ribozyme. In the latter case, differences in catalytic behavior of the N76 mutants coincide instead with changes in average conformation and modified dynamic cross-correlations in the catalytic core, as shown by complementary terbium(III) footprinting assays and MD simulations (Figures 6 and 8A), respectively. More specifically, larger conformational flexibility and dynamics in the catalytic core during MD simulation coincide with higher catalytic activity (Figure 8B).

The surprising difference in the dependence of catalytic activity on the identity of the N76 base between the transand cis-acting HDV ribozymes may be related to their distinct sequence composition and folding. While both constructs follow the same reaction pathway, the cis-acting ribozyme has an $\sim 20$-fold higher cleavage rate constant than the trans-acting version. This may be related to the fact that, according to terbium(III) footprinting assays, the short P1.1 stem appears to form only in the reaction precursor of the cis-acting, but not that of the trans-acting ribozyme. ${ }^{18,29}$ As P1.1 is essential for catalysis, ${ }^{6}$ a conformational change from a P1.1-free ground-state conformation to a catalytically active P1.1-containing conformation may be (partially) ratelimiting for the trans-, but not the cis-acting ribozyme. An incompletely formed P1.1 stem in the trans- compared with the cis-acting ribozyme may also contribute to a less tightly folded catalytic core and a more pronounced dependency on G76 to make at least a partial tertiary contact with the major groove of the P1 stem and thus stabilize the core. It is plausible that in the cis-acting genomic ribozyme less base-specific and more transient N76 interactions with the backbone of the P1 stem, as observed in our MD simulations, suffice to stabilize the structure.

An extensive mutagenesis study of the single-stranded regions of the cis-acting genomic HDV ribozyme distinguished between mutations that affect catalysis and those that promote alternative folding of the RNA into inactive conformations. ${ }^{16}$ Compared with this previous study, our genomic HDV ribozyme has shorter $5^{\prime}$ and $3^{\prime}$ ends and cleavage was optimized by following an extensive annealing protocol. ${ }^{24}$ G76 renders the ribozyme highly active and promotes folding into an active conformation (as indicated by its high extent of cleavage), consistent with its dominance in almost all clinical isolates of the genomic HDV ribozyme. ${ }^{32}$ The fact that only one other mutant, G76A, was identified in a clinical isolate may relate to the comparable catalytic activity found for the wt and G76A variants (Figure 5, Table II). By contrast, pyrimidines in position 76 behave very differently. While cleavage of the G76U mutant was observed to be faster than that of the wt, the final extent of RNA cleavage is lower in this case, suggesting enhanced misfolding (Figure 5, Table II). The G76C mutant, by contrast, exhibits a comparable extent of cleavage as the wt, but at a 2.4-fold slower rate constant.

Terbium(III)-mediated footprinting demonstrates that significant differences in scission intensity between the N76 variants are confined to the joiner $\mathrm{J} 4 / 2$ sequence, providing strong evidence that they all share the overall structure of the wt (Figure 6). Previous studies of the stability of the backbone of unstructured RNA reported that the sequences $5^{\prime}$ UpA- $3^{\prime}$ and $5^{\prime}-\mathrm{CpA}-3^{\prime}$ are $>100$ - and up to 35 -fold, respectively, less chemically stable than any other dinucleotide steps. ${ }^{33,34}$ The joiner J4/2 sequences of the G76A, G76C, and G76U mutant ribozymes all contain such dinucleotide steps. The $5^{\prime}$-C75pA76- $3^{\prime}$ dinucleotide step in the G76A mutant, for example, may therefore be expected to lead to a more intensive peak $3^{\prime}$ of $\mathrm{C} 75$ than the wt sequence, yet the scission pattern of the G76A mutant is similar to that of the wt ribozyme (Figure 6). Similarly, one may expect a more intense peak $3^{\prime}$ of $\mathrm{C} 76$ in the $5^{\prime}-\mathrm{C} 76 \mathrm{pA} 77-3^{\prime}$ sequence of the G76C mutant, however, the A77 peak is higher than that of C76 (Figure 6). Only the fact that we observe enhanced scission $3^{\prime}$ of U76 in the $5^{\prime}$-U76pA77-3' dinucleotide step of the G76U mutant may in part be influenced by the lower chemical stability of this sequence in the context of an unstructured RNA. Nevertheless, the tight double-nested pseudoknot fold of the cis-acting HDV ribozyme seems to largely suppress the nonspecific backbone scission effects found in unstructured RNA so that differences in our terbium(III) scission patterns primarily reflect changes in average conformation of the trefoil turn in response to the identity of N76. The observed correlation between the terbium(III) scission patterns and the reaction rate constants therefore are likely significant, where a wt-like scission pattern of the G76A mutant correlates with a close-to wt activity and where pyrimidines in position 76 show both distinct terbium(III) footprinting profiles and catalytic activities.

Naturally, equilibrium footprinting experiments cannot provide the detailed atomistic information that MD simulations do, but the two techniques are complementary. For example, our experimental observation of distinct terbiu$\mathrm{m}$ (III) footprinting patterns and catalytic activities of the N76 variants correlates with the fact that these variants show distinct conformational substates in our MD simulations, characterized by stable $\mathrm{H}$-bond interactions of N76 with 
either bases or the backbone of the P1 stem (Figure 7), which typically coincide with a less stable C75:A77 stack (Figure $8 \mathrm{~B})$. While our identification of these various substates is based on $\sim 136$ ns total simulation time for the wt and $\sim 40$ ns for each of the mutants, it should be noted that, as for any simulated biopolymer, conformational sampling is incomplete and force fields and especially their description of divalent cations are relatively crude approximations. ${ }^{20,21,35}$ This limitation becomes particularly evident when comparing with equilibrium structure probing techniques, which by their very nature average over a (nearly) infinite number of molecules and length of time. To cope with the sampling limitation of $\mathrm{MD}$, we ran several simulations for any given N76 variant; in addition, we attempted to employ advanced simulation techniques such as LES on the wild-type ribozyme, which however did not converge. An alternative computational technique for bridging divergent simulation and experimental time scales is umbrella sampling, which increases sampling by calculating the potential of mean force along a defined reaction coordinate while taking into account results obtained in previous steps. To obtain a reliable thermodynamic free-energy profile and identify conformational transition barrier(s), a good reaction coordinate has to be chosen, a nontrivial task, and a sufficiently dense grid of calculation points along the coordinate has to be pursued to ensure convergence, leading to a substantial computational expense. Conversely, photo-induced crosslinking could be pursued to experimentally trap transient conformations observed in our MD simulations. Such approaches, however, go beyond the scope of the work presented here.

The particular appeal of our approach here is the combination of MD simulations with complementary structure and function probing techniques such as footprinting assays and computationally inspired second-site mutagenesis experiments. By going back and forth between theory and experiment, our studies demonstrate a powerful synergy in revealing the conformational sampling of a ribozyme catalytic core in solution, as is essential for its enzymatic function. In the case of the cis-acting genomic HDV ribozyme this synergistic approach provides a more realistic picture of the structurally dynamic, catalytically involved trefoil motif than is possible by static crystal structures alone, which in part turn out to be conformationally constrained by crystal lattice contacts.

\section{MATERIALS AND METHODS}

\section{Initial Structures}

Our simulations started from the crystal structures of the precursor (PDB ID 1SJ3; $2.20 \AA$ resolution) ${ }^{9}$ and $3^{\prime}$ product forms (PDB ID
$1 \mathrm{CX} 0 ; 2.3 \AA$ resolution $)^{11}$ of the HDV ribozyme as previously described. $^{20,21}$ To obtain the initial structures of the G76 mutants, G76 in the $3^{\prime}$ product form was modified to A76, C76, and U76 using InsightII.

\section{Simulations}

All MD simulations were carried out using the AMBER7.0 program package $^{36}$ with the parm99 Cornell et al. force field. ${ }^{37-39}$ The RNA was solvated in a rectangular box of TIP3P waters ${ }^{40}$ extended to a distance of $\geq 10 \AA$ from any solute atom. $\mathrm{Mg}^{2+}$ cations were taken from the respective PDB file. The simulated system was charge neutralized by the addition of sodium cations ${ }^{41}$ initially placed by the LeaP module at points of favorable electrostatic potential close to the RNA. This corresponds to an ion concentration of $\sim 0.1 \mathrm{M}$. The Sander module of AMBER7.0 was used for the equilibration and production simulations using standard protocols. ${ }^{20,21}$ The particle mesh Ewald method ${ }^{42}$ was applied with a heuristic pair list update, using a 2.0-^̊ nonbonded pair list buffer and a $9.0 \AA$ cutoff. A charge grid spacing of close to $1 \AA$ and a cubic interpolation scheme were used. The production runs were carried out at $300 \mathrm{~K}$ with constantpressure boundary conditions using the Berendsen temperature coupling algorithm ${ }^{43}$ with a time constant of 1.0 ps. SHAKE ${ }^{44}$ was applied in the simulations with a tolerance of $10^{-8}$ to constrain bonds involving hydrogens.

\section{Analysis of MD Trajectories}

MD trajectories were analyzed using the carnal and ptraj modules of the AMBER7.0 package. Specific structures were visualized using the programs $\mathrm{VMD}^{45}$ and InsightII (Accelrys). The occupancy criterion for a $\mathrm{H}$-bond was defined as the time in percent during which the hydrogen bond distance between the heavy atoms (donor (D) and acceptor (A)) was $\leq 3.0 \AA$ and the H-bond angle $\mathrm{D}-\mathrm{H}-\mathrm{A}$ was $\geq 120^{\circ}$. Time trajectories of heavy-atom distances were then depicted as density plots using the program Mathematica 5 or as standard line plots using Origin 7.0. The ptraj module of AMBER-8 was used to obtain cross-correlation matrices whose output data were visualized using Mathematica 5.

\section{Preparation of RNA Oligonucleotides}

RNA oligonucleotides for the three-strand trans-acting HDV ribozyme depicted in Figure 4A were purchased with $2^{\prime}$-protection groups from the HHMI Biopolymer/Keck Foundation Biotechnology Resource Laboratory at the Yale University School of Medicine (New Haven, CT) and were deprotected as described. ${ }^{8,46,47}$ Deprotected RNA was purified by denaturing ( $20 \%$ polyacrylamide, $8 \mathrm{M}$

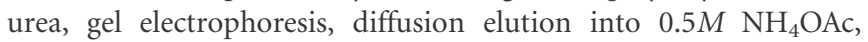
$0.1 \%$ SDS, and $0.1 \mathrm{mM}$ EDTA) overnight at $4^{\circ} \mathrm{C}$, chloroform extraction, ethanol precipitation, and C8 reverse-phase HPLC with a linear acetonitrile gradient in triethylammonium acetate, as described previously. ${ }^{8,46,47}$ RNA concentrations were calculated from their absorption at $260 \mathrm{~nm}$.

The cis-acting precursor and product forms of the genomic HDV ribozyme in Figure 5A were generated by run-off transcription from a double-stranded, PCR amplified template that encoded an upstream T7 promoter. Transcription reactions contained $40 \mathrm{mM}$ Tris- $\mathrm{HCl}$ ( $\mathrm{pH}$ 7.5), $15 \mathrm{mM} \mathrm{MgCl}$, $5 \mathrm{mM}$ dithiothreitol, $2 \mathrm{mM}$ spermidine, $4 \mathrm{mM}$ 
each rNTP, 5 units $/ \mathrm{ml}$ of inorganic pyrophosphatase, and $0.1 \mathrm{mg} / \mathrm{ml}$ of T7 RNA polymerase and were incubated at $37^{\circ} \mathrm{C}$ for $2.5 \mathrm{~h}$. Protein was removed by phenol/chloroform extractions and the RNA was concentrated using Centricon YM-3 (3000-Da cutoff) filters. The full-length transcripts of the product form were isolated after electrophoresis on denaturing, $8 M$ urea, $8 \%(\mathrm{w} / \mathrm{v})$ polyacrylamide gels by UV shadowing, diffusion elution of small gel slices into a 4 $\mathrm{ml}$ solution of $1 \mathrm{mM}$ EDTA. RNA was recovered by ethanol precipitation and was then dissolved in autoclaved doubly deionized water and stored at $-20^{\circ} \mathrm{C}$.

For self-cleavage reactions, the radiolabeled precursor HDV ribozyme was transcribed as described earlier, except that $0.4 \mathrm{mCi}$ of $\left[\alpha_{-}{ }^{32} \mathrm{P}\right]$ GTP were added to the reaction mixture. The transcription reaction was incubated for $24 \mathrm{~h}$ at $10^{\circ} \mathrm{C}$ and the RNA was fractionated by electrophoresis on a denaturing, $8 M$ urea, $8 \%(w / v)$ polyacrylamide gel. Uncleaved precursor RNA was located by autoradiography, excised, eluted into a $2 \mathrm{ml}$ solution of $1 \mathrm{mM}$ EDTA ( $\mathrm{pH}$ 8.0) overnight at $4{ }^{\circ} \mathrm{C}$ and recovered by ethanol precipitation. The radiolabeled RNA was stored in $0.1 \mathrm{mM}$ EDTA ( $\mathrm{pH} 8.0$ ) at $-20^{\circ} \mathrm{C}$. The EDTA solution was prior incubated with a bed of Chelex100 to remove residual divalent cations.

\section{Cleavage Assays}

Cleavage activities of trans-acting HDV ribozymes with mutations at positions 2, 36, and 76 were determined with radiolabeled substrate (Figure 4). $5^{\prime}-{ }^{32} \mathrm{P}$-labeled substrate was generated by phosphorylation with T4 polynucleotide kinase and $\left[\gamma_{-}^{32} \mathrm{P}\right]$ ATP, followed by desalting using a CentriSep spin column (Princeton Separations). All cleavage reactions on the trans-acting ribozyme were conducted under single-turnover (pre-steady-state) conditions. Standard conditions were $40 \mathrm{mM}$ Tris- $\mathrm{HCl}(\mathrm{pH} 7.5)$ and $11 \mathrm{mM} \mathrm{MgCl}_{2}$ at $37^{\circ} \mathrm{C}$. Ribozyme was preannealed from strand $\mathrm{A}$ and twice the concentrations of strand $\mathrm{B}$ in standard buffer, by heating to $70^{\circ} \mathrm{C}$ for $2 \mathrm{~min}$ and cooling to room temperature over the course of $5 \mathrm{~min}$. After preincubation for $15 \mathrm{~min}$ at $37^{\circ} \mathrm{C}$, a trace $(<4 \mathrm{nM})$ concentration of $5^{\prime}-{ }^{32} \mathrm{P}$-labeled substrate in standard buffer was added to a final concentration of 50-6000 nM ribozyme (based on the strand A concentration). Aliquots $(5 \mu \mathrm{l})$ were taken at appropriate time intervals, and the reaction was quenched with $10 \mu \mathrm{l}$ of $80 \%$ formamide, $0.025 \%$ xylene cyanol, $0.025 \%$ bromophenol blue, and $50 \mathrm{mM}$ EDTA. The $5^{\prime}$-cleavage product was separated from the uncleaved substrate by denaturing, $8 \mathrm{M}$ urea, $20 \%$ polyacrylamide gel electrophoresis, and was quantified and normalized to the sum of the substrate and product bands using a PhosphorImager Storm 840 instrument with ImageQuaNT software (Molecular Dynamics). Time traces of product formation were fit with the single-exponential first-order rate equation $y=y_{0}+A_{1}\left(1-e^{-t / \tau_{1}}\right)$, employing Marquardt-Levenberg nonlinear least-squares regression (Microcal Origin 7.0), where $A_{1}$ is the amplitude and $1 / \tau_{1}$ the pseudo-firstorder rate constant $k_{\mathrm{obs}}$. Duplicates of at least eight different ribozyme concentrations $([\mathrm{Rz}])$ were used to extract the catalytic rate constant $k_{\text {cleav }}$ under standard conditions, by fitting the ribozyme concentration dependence of $k_{\mathrm{obs}}$ to the simple binding equation:

$$
k_{\mathrm{obs}}=k_{\text {cleav }}\left([\mathrm{Rz}] /\left([\mathrm{Rz}]+\mathrm{Rz}_{1 / 2}\right)\right)
$$

similar to previously described procedures. ${ }^{8} \mathrm{Rz}_{1 / 2}$ describes the ribozyme half-titration point associated with catalysis. Errors in fit parameters were obtained from the standard deviation of the fit.

Radiolabeled precursor forms of the cis-acting ribozymes with mutations at position 76 and in the P1 stem (Figure 5) were heated to $90^{\circ} \mathrm{C}$ for $2 \mathrm{~min}$ in a buffer containing $5 \mathrm{mM}$ Tris- $\mathrm{HCl}(\mathrm{pH} 7.5$ ), $0.5 \mathrm{mM}$ spermidine, and $1 \mathrm{~m} M$ EDTA. The precursors were then preincubated at $37^{\circ} \mathrm{C}$ for $10 \mathrm{~min}$, after which the reactions were adjusted to the final $\mathrm{pH}$ with a buffer containing $25 \mathrm{mM}$ acetic acid, $25 \mathrm{mM}$ Mes, and $50 \mathrm{mM}$ Tris- $\mathrm{HCl}$ ( $\mathrm{pH}$ 7.5). These mixtures were incubated for an additional $5 \mathrm{~min}$ at $37^{\circ} \mathrm{C}$ and then aliquots $(4 \mu \mathrm{l})$ distributed to the reaction wells at $22^{\circ} \mathrm{C}$. Cleavage at $22^{\circ} \mathrm{C}$ was initiated by addition of an equal volume of a solution containing 22 $\mathrm{mM} \mathrm{MgCl} 2,0.2 \mathrm{mM}$ spermidine, and $0.4 \mathrm{mM}$ EDTA. Cleavage kinetics were followed by quenching reaction aliquots at appropriate times with $8 \mu \mathrm{l}$ of $80 \%(\mathrm{v} / \mathrm{v})$ formamide, $0.025 \%(\mathrm{w} / \mathrm{v})$ xylene cyanol, $0.025 \%(\mathrm{w} / \mathrm{v}$ ) bromophenol blue, and $50 \mathrm{mM}$ EDTA. The reaction product was separated from the precursor by gel electrophoresis under denaturing conditions ( $8 M$ urea, $8 \%(\mathrm{w} / \mathrm{v})$ polyacrylamide gels), quantified and analyzed as described earlier.

\section{Terbium(III)-Mediated Footprinting}

The highest purity terbium(III) chloride (99.9\%) was purchased from Sigma-Aldrich. $\mathrm{TbCl}_{3}$ stock solutions at $100 \mathrm{mM}$ were prepared in $5 \mathrm{mM}$ sodium cacodylate ( $\mathrm{pH}$ 5.5) and stored in small aliquots at $-20^{\circ} \mathrm{C}$ to prevent formation of insoluble hydroxide species at higher $\mathrm{pH}$, as previously described..$^{25,30}$

To observe the slow backbone scission mediated by the deprotonated $\mathrm{Tb}(\mathrm{OH})(\mathrm{aq})^{2+}$ species, the purified self-cleaved product forms of the various genomic HDV ribozymes were $\left(5^{\prime}-{ }^{32} \mathrm{P}\right)$-phosphorylated with T4 polynucleotide kinase and $\left[\gamma-{ }^{32} \mathrm{P}\right] \mathrm{ATP}$ and repurified by denaturing, $8 M$ urea, $15 \%(\mathrm{w} / \mathrm{v})$ polyacrylamide gel electrophoresis, followed by diffusion elution into $1 \mathrm{ml}$ of $0.1 \mathrm{mM}$ EDTA ( $\mathrm{pH}$ 8.0), and ethanol precipitation, as described previously. ${ }^{29,30,48}$ The radiolabeled RNA $(180,000 \mathrm{cpm}$ per $10 \mu \mathrm{l}$ reaction volume) was preannealed in buffer ( $5 \mathrm{mM}$ Tris- $\mathrm{HCl}, \mathrm{pH} 7.5,0.5$ $\mathrm{m} M$ spermidine), denatured at $90^{\circ} \mathrm{C}$ for $2 \mathrm{~min}$, and incubated at $37^{\circ} \mathrm{C}$ for $10 \mathrm{~min}$. The final $\mathrm{pH}$ was adjusted with a buffer containing $25 \mathrm{mM}$ acetic acid, $25 \mathrm{mM}$ Mes, and $50 \mathrm{mM}$ Tris- $\mathrm{HCl}$ (pH 7.5). To fold the tertiary structure of the RNA, $\mathrm{Mg}^{2+}$ (11 mM final concentration) with spermidine $(0.1 \mathrm{mM}$ final concentration) were added, immediately followed by the addition of an appropriate $\mathrm{Tb}^{3+}$ stock solution ( $1 \mathrm{mM}$ final concentration) and incubation for an $1 \mathrm{~h}$ at $37^{\circ} \mathrm{C}$. The scission reaction was stopped by adding EDTA ( $\left.\mathrm{pH} 8.0\right)$ to a final concentration of $50 \mathrm{mM}$ and by overnight ethanol precipitation at $-20^{\circ} \mathrm{C}$. The precipitated RNA was redissolved in urea loading buffer ( $80 \%$ formamide, $0.025 \%$ xylene cyanol, $0.025 \%$ bromophenol blue, $9 M$ urea) and analyzed on an $8 M$ urea, wedged $15 \%$ polyacrylamide sequencing gel, alongside sequencing ladders from partial digestion with G-specific RNase T1 and alkaline hydrolysis as described. ${ }^{29,30,48}$ Product bands were directly visualized using autoradiography and quantified using a PhosphorImager Storm 840 with ImageQuaNT software (Molecular Dynamics).

\section{REFERENCES}

1. Lai, M. M. Annu Rev Biochem 1995, 64, 259-286.

2. Perrotta, A. T.; Been, M. D. Nucleic Acids Res 1990, 18, 68216827. 
3. Perrotta, A. T.; Been, M. D. Nature 1991, 350, 434-436.

4. Been, M. D.; Wickham, G. S. Eur J Biochem 1997, 247, 741753.

5. Wadkins, T. S.; Been, M. D. Nucleic Acids Res 1997, 25, 40854092.

6. Wadkins, T. S.; Perrotta, A. T.; Ferre-D’Amare, A. R.; Doudna, J. A.; Been, M. D. RNA 1999, 5, 720-727.

7. Narlikar, G. J.; Herschlag, D. Annu Rev Biochem 1997, 66, 19 59.

8. Pereira, M. J.; Harris, D. A.; Rueda, D.; Walter, N. G. Biochemistry 2002, 41, 730-740.

9. Ke, A.; Zhou, K.; Ding, F.; Cate, J. H.; Doudna, J. A. Nature 2004, 429, 201-205.

10. Shih, I. H.; Been, M. D. Annu Rev Biochem 2002, 71, 887-917.

11. Ferre-D'Amare, A. R.; Doudna, J. A. J Mol Biol 2000, 295, 541556.

12. Ferre-D’Amare, A. R.; Zhou, K.; Doudna, J. A. Nature 1998, 395, 567-574.

13. Perrotta, A. T.; Shih, I.; Been, M. D. Science 1999, 286, 123-126.

14. Nakano, S.; Chadalavada, D. M.; Bevilacqua, P. C. Science 2000, 287, 1493-1497.

15. Das, S. R.; Piccirilli, J. A. Nat Chem Biol 2005, 1, 45-52.

16. Tanner, N. K.; Schaff, S.; Thill, G.; Petit-Koskas, E.; CrainDenoyelle, A. M.; Westhof, E. Curr Biol 1994, 4, 488-498.

17. Harris, D. A.; Rueda, D.; Walter, N. G. Biochemistry 2002, 41, 12051-12061.

18. Jeong, S.; Sefcikova, J.; Tinsley, R. A.; Rueda, D.; Walter, N. G. Biochemistry 2003, 42, 7727-7740.

19. Tinsley, R. A.; Harris, D. A.; Walter, N. G. Biochemistry 2004, 43, 8935-8945.

20. Krasovska, M. V.; Sefcikova, J.; Špacková, N.; Šponer, J.; Walter, N. G. J Mol Biol 2005, 351, 731-748.

21. Krasovska, M. V.; Sefcikova, J.; Reblova, K.; Schneider, B.; Walter, N. G.; Sponer, J. Biophys J 2006, 91, 626-638.

22. Gondert, M. E.; Tinsley, R. A.; Rueda, D.; Walter, N. G. Biochemistry 2006, 45, 7563-7573.

23. Tinsley, R. A.; Harris, D. A.; Walter, N. G. J Am Chem Soc 2003, 125, 13972-13973.

24. Wadkins, T. S.; Shih, I.; Perrotta, A. T.; Been, M. D. J Mol Biol 2001, 305, 1045-1055.

25. Walter, N. G.; Yang, N.; Burke, J. M. J Mol Biol 2000, 298, 539555.

26. Hargittai, M. R.; Musier-Forsyth, K. RNA 2000, 6, 1672-1680.

27. Hargittai, M. R.; Mangla, A. T.; Gorelick, R. J.; Musier-Forsyth, K. J Mol Biol 2001, 312, 985-997.

28. Sigel, R. K. O.; Pyle, A. M. Met Ions Biol Syst 2003, 40, 477-512.

29. Harris, D. A.; Tinsley, R. A.; Walter, N. G. J Mol Biol 2004, 341, 389-403.
30. Harris, D. A.; Walter, N. G. In Handbook of RNA Biochemistry; Hartmann, R. K.; Bindereif, A.; Schön, A.; Westhof, E., Eds.; Wiley-VCH: Weinheim, 2005; pp 205-213.

31. Fadrna, E.; Spackova, N.; Stefl, R.; Koca, J.; Cheatham, T. E., III; Sponer, J. Biophys J 2004, 87, 227-242.

32. Wadkins, T. S.; Been, M. D. Cell Mol Life Sci 2002, 59, 112-125.

33. Bibillo, A.; Figlerowicz, M.; Ziomek, K.; Kierzek, R. Nucleosides Nucleotides Nucleic Acids 2000, 19, 977-994.

34. Kaukinen, U.; Lyytikainen, S.; Mikkola, S.; Lonnberg, H. Nucleic Acids Res 2002, 30, 468-474.

35. McDowell, S. E.; Spackova, N.; Sponer, J.; Walter, N. G. Biopolymers 2007, 85, 169-184.

36. Case, D. A.; Pearlman, D. A.; Caldwell, J. W.; Cheatham, T. E., III; Wang, J.; Ross, W. S.; Simmerling, C. L.; Darden, T. A.; Merz, K. M.; Stanton, R. V.; Cheng, A. L.; Vincent, J. J.; Crowley, M.; Tsui, V.; Gohlke, H.; Radmer, R. J.; Duan, Y.; Pitera, J.; Massova, I.; Seibel, G. L.; Singh, U. C.; Weiner, P. K.; Kollman, P. A. AMBER 7; University of California San Francisco: San Francisco, 2002.

37. Cheatham, T. E., III; Cieplak, P.; Kollman, P. A. J Biomol Struct Dyn 1999, 16, 845-862.

38. Cornell, W. D.; Cieplak, P.; Bayly, C. I.; Gould, I. R.; Merz, K. M.; Ferguson, D. M.; Spellmeyer, D. C.; Fox, T.; Caldwell, J. W.; Kollman, P. A. J Am Chem Soc 1995, 117, 5179-5197.

39. Wang, J. M.; Cieplak, P.; Kollman, P. A. J Comput Chem 2000, 21, 1049-1074.

40. Jorgensen, W. L.; Chandrasekhar, J.; Madura, J. D.; Impey, R. W.; Klein, M. L. J Chem Phys 1983, 79, 926-935.

41. Aqvist, J. J Phys Chem 1990, 94, 8021-8024.

42. Essmann, U.; Perera, L.; Berkowitz, M. L.; Darden, T.; Lee, H.; Pedersen, L. G. J Chem Phys 1995, 103, 8577-8593.

43. Berendsen, H. J. C.; Postma, J. P. M.; Vangunsteren, W. F.; Dinola, A.; Haak, J. R. J Chem Phys 1984, 81, 3684-3690.

44. Ryckaert, J. P.; Ciccotti, G.; Berendsen, H. J. C. J Comput Chem 1977, 23, 327-341.

45. Humphrey, W.; Dalke, A.; Schulten, K. J Mol Graph 1996, 14, 33-38.

46. Walter, N. G. Methods 2001, 25, 19-30.

47. Walter, N. G. Curr Protocols Nucleic Acid Chem 2002, 11.10, 11.10.11-11.10.23.

48. Harris, D. A.; Walter, N. G. Curr Protocols Nucleic Acid Chem 2003, 6.8, 6.8.1-6.8.8.

Reviewing Editor: J. Andrew McCammon 